\title{
Republic of Yemen: Statistical Appendix
}

This Statistical Appendix for the Republic of Yemen was prepared by a staff team of the International Monetary Fund as background documentation for the periodic consultation with the member country. It is based on the information available at the time it was completed on February 28, 2005. The views expressed in this document are those of the staff team and do not necessarily reflect the views of the government of the Republic of Yemen or the Executive Board of the IMF.

The policy of publication of staff reports and other documents by the IMF allows for the deletion of market-sensitive information.

To assist the IMF in evaluating the publication policy, reader comments are invited and may be sent by e-mail to publicationpolicy@imf.org.

Copies of this report are available to the public from

International Monetary Fund • Publication Services

$70019^{\text {th }}$ Street, N.W. • Washington, D.C. 20431

Telephone: (202) 623-7430 • Telefax: (202) 623-7201

E-mail: publications@imf.org•Internet: http://www.imf.org

Price: $\$ 15.00$ a copy

\section{International Monetary Fund Washington, D.C.}





\title{
INTERNATIONAL MONETARY FUND
}

\author{
REPUBLIC OF YEMEN
}

\begin{abstract}
Statistical Appendix
Prepared by a staff team consisting of Mr. S. Chami (head), Mr. N. Ben Ltaifa, Ms. A. Ivanova, Mr. Y. Abdih (all MCD), Ms. Jacobs (FAD), and Mr. Ahmed (PDR)

Approved by the Middle East and Central Asia Department
\end{abstract}

February 28, 2005

Contents

Page

Tables

1. Social and Demographic Indicators, 1998-2003 ……….........................................

2. Selected Economic and Financial Indicators, 2000-03 .............................................

3. Sectoral Origin of Gross Domestic Product at Current Prices, 1998-2003 .................6

4. Sectoral Origin of Gross Domestic Product at Constant Prices, 1998-2003 ...............7

5. Use of Resources at Current Prices, 1998-2003 …………......................................

6. Distribution of Employment (Age 15 Years and Over) by Economic Activity, 1999-2003.

7. Social Fund for Development-Accumulated Investment, Disbursement, Number of Expected Beneficiaries, and Job Opportunities, December 31, 2001; December 31, 2002; September 30, 2003; and December 31, 2004 According to Sector.

8. Social Fund for Development - Total Investment and Disbursement Up to September 30, 2004

9. Distribution of Labor Force by Employment Status, Age Group (+10 Years), and Gender, 1994, 1999, and 2002

10. Students' Enrollment and Graduation from Universities in 2001/02 and 2002/03 ....14

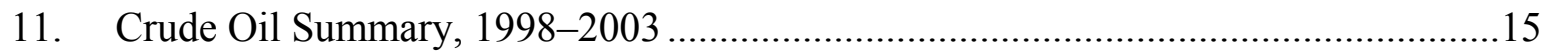

12. Domestic Consumption of Petroleum Products, 1998-2003 …………………..........16

13. Output of Industrial Products, 1998-2003 ……….............................................17

14. Production, Area, and Yield of Major Crops, 1998-2003 …………........................18

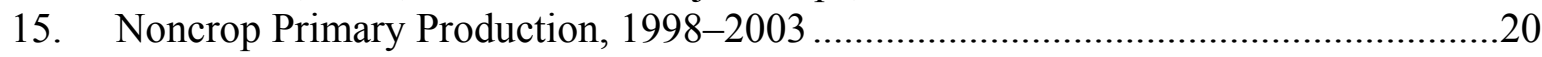

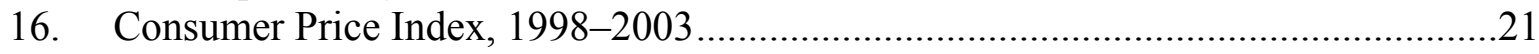

17. Domestic Retail Prices for Petroleum Products and Electricity, 1998-2003 .............22

18. Summary of Central Government Finance, 1998-2003 ……………………...........23

19. Composition of Central Government Revenues, 1998-2003 ……...........................25

20. Composition of Central Government Expenditures, 1998-2003 ................................27

21. Current and Capital Transfers to Public Enterprises, 1998-2001 .............................29 


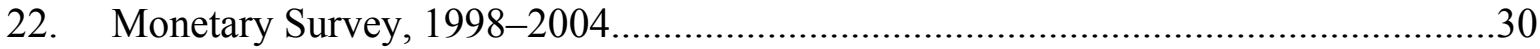

23. Factors Affecting Domestic Liquidity, 1998-2004 …........................................32

24. Balance Sheet of the Central Bank, 1998-2004 …...................................................34

25. Consolidated Balance Sheet of Commercial Banks, 19972004 ................................36

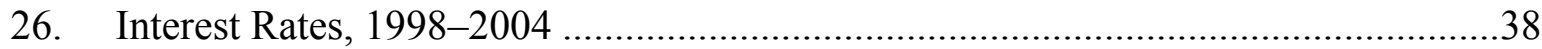

27. Distribution of Commercial Bank Credit to the Nongovernment Sector,

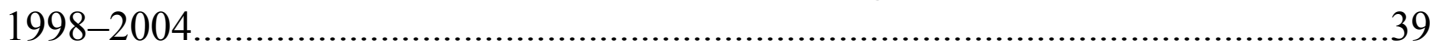

28. Commercial and Specialized Banks, at end-2004 ..............................................40

29. Indicators of Banking System Financial Soundness, 1999-2003 ...........................41

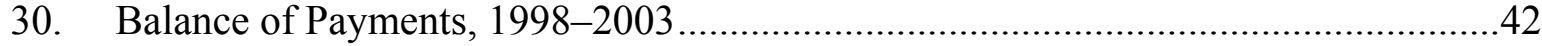

31. Composition of Exports and Re-exports, 1998-2003 ….......................................43

32. Direction of Exports and Re-exports, 1998-2003 .............................................44

33. Composition of Imports, 1998-2003 .............................................................45

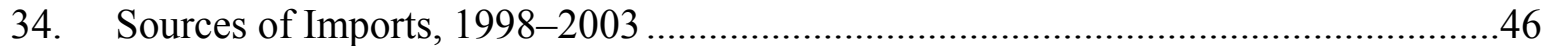

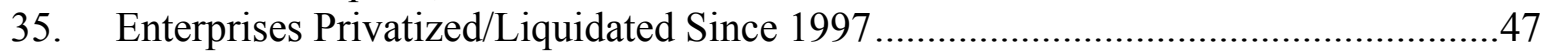


Table 1. Republic of Yemen: Social and Demographic Indicators, 1998-2003

\begin{tabular}{|c|c|c|c|c|c|c|}
\hline & 1998 & 1999 & 2000 & 2001 & 2002 & 2003 \\
\hline \multicolumn{7}{|l|}{ Socio-demographic indicators } \\
\hline GNP per capita (in U.S. dollars) & 342 & 394 & 495 & 478 & 482 & 523 \\
\hline Total population (in millions) & 16.5 & 17.1 & 17.6 & 18.2 & 18.9 & 19.5 \\
\hline Population growth rate (in percent) & 2.9 & 3.3 & 3.2 & 3.4 & 3.6 & 3.5 \\
\hline Total fertility rate & $\ldots$ & 6.2 & 6.2 & 6.2 & 6.2 & 6.2 \\
\hline \multicolumn{7}{|l|}{ Age dependency ratio } \\
\hline (Dependants to working-age population) & 1.1 & 1.0 & 1.1 & 1.0 & $\ldots$ & $\ldots$ \\
\hline Urban population (in percent) & 24.3 & 24.5 & 24.7 & 25.0 & 26.5 & 26.5 \\
\hline \multicolumn{7}{|l|}{ Labor participation rate } \\
\hline (In percent of population aged 14-64) & $\ldots$ & 46.0 & 46.0 & $\ldots$ & $\ldots$ & $\ldots$ \\
\hline $\begin{array}{l}\text { Female labor participation rate } \\
\text { (In percent of total population) }\end{array}$ & 28.0 & 21.8 & 22.0 & 22.6 & 22.5 & 24.0 \\
\hline \multicolumn{7}{|l|}{ Health } \\
\hline Life expectancy at birth (in years) & $\ldots$ & 56.0 & 57.0 & 57.0 & 58.0 & 58.0 \\
\hline Infant mortality rate (per 1,000 live births) & $\ldots$ & 79.0 & 67.0 & $\ldots$ & $\ldots$ & $\ldots$ \\
\hline Under five mortality rate (per 1,000 live births) & $\ldots$ & 97.0 & 95.0 & $\ldots$ & $\ldots$ & 83.0 \\
\hline $\begin{array}{l}\text { Immunized: DPT, under } 12 \text { months } \\
\text { (In percent of age group) }\end{array}$ & 68.0 & 72.0 & $\ldots$ & 76.0 & $\ldots$ & $\ldots$ \\
\hline Access to safe water (percent of population) & $\ldots$ & $\ldots$ & 49.0 & 49.6 & 49.7 & 50.0 \\
\hline \multicolumn{7}{|l|}{ Education } \\
\hline Adult literacy rate & $\ldots$ & 45.0 & 46.0 & 46.3 & $\ldots$ & 47.0 \\
\hline
\end{tabular}

Sources: Central Statistics Organization, Statistical Year Book; and IBRD, World Development Indicators database. 
Table 2. Republic of Yemen: Selected Economic and Financial Indicators, 2000-2003

\begin{tabular}{|c|c|c|c|c|}
\hline & 2000 & 2001 & 2002 & 2003 \\
\hline & \multirow{2}{*}{\multicolumn{4}{|c|}{ (Change in percent) }} \\
\hline Production and prices & & & & \\
\hline Nominal GDP at market prices & 31.3 & 4.5 & 9.0 & 16.1 \\
\hline Real GDP at market prices & 4.4 & 4.6 & 3.9 & 3.1 \\
\hline Real non-oil GDP & 3.5 & 5.2 & 4.6 & 4.0 \\
\hline Real oil GDP & 9.4 & 1.3 & 0.4 & -1.8 \\
\hline GDP deflator & 25.7 & -0.1 & 4.9 & 12.5 \\
\hline Core consumer price index (annual average) 1/ & 10.0 & 10.7 & 6.8 & 11.9 \\
\hline Core consumer price index (12-month) $1 /$ & 7.6 & 14.6 & 4.5 & 12.1 \\
\hline Crude oil production (1,000 barrels/day) & 434 & 434 & 433 & 425 \\
\hline \multicolumn{5}{|l|}{ Crude export oil price (weighted average, } \\
\hline \multirow[t]{2}{*}{ U.S. dollar/barrel) $2 /$} & 28.2 & 23.0 & 24.6 & 27.9 \\
\hline & \multicolumn{4}{|c|}{ (In percent of GDP) } \\
\hline \multicolumn{5}{|l|}{ Government finance } \\
\hline Total revenue and grants & 39.2 & 35.3 & 33.6 & 33.1 \\
\hline Oil revenue & 27.9 & 25.3 & 22.3 & 23.6 \\
\hline Non-oil revenue & 10.0 & 9.8 & 9.7 & 9.1 \\
\hline Grants & 1.3 & 0.3 & 1.6 & 0.4 \\
\hline Total expenditure & 31.2 & 32.8 & 34.8 & 38.2 \\
\hline Current & 25.8 & 25.2 & 27.7 & 28.6 \\
\hline Development & 5.4 & 7.5 & 7.1 & 9.7 \\
\hline Overall balance including grants (commitment basis) & 8.0 & 2.6 & -1.2 & -5.2 \\
\hline \multirow{3}{*}{$\begin{array}{l}\text { Primary non-oil fiscal balance (commitment, including } \\
\text { grants, excluding oil and gas revenues) }\end{array}$} & & & & \\
\hline & -17.6 & -20.5 & -21.5 & -26.9 \\
\hline & \multicolumn{4}{|c|}{ (12-month change in percent) } \\
\hline \multicolumn{5}{|l|}{ Monetary data } \\
\hline Broad money & 25.1 & 18.7 & 18.0 & 20.0 \\
\hline Credit to private sector & 21.3 & 25.8 & 14.3 & 26.3 \\
\hline Benchmark deposit interest rate (percent per annum) & 13.0 & 13.0 & 13.0 & 13.0 \\
\hline \multirow[t]{2}{*}{ Velocity (Non-oil GDP/M2) } & 2.4 & 2.2 & 2.1 & 2.0 \\
\hline & \multicolumn{4}{|c|}{ (In millions of U.S. dollars) } \\
\hline \multicolumn{5}{|l|}{ External sector } \\
\hline Exports, f.o.b. & 3,805 & 3,302 & 3,584 & 3,923 \\
\hline Of which: crude oil & 3,423 & 2,895 & 3,134 & 3,417 \\
\hline Imports, f.o.b. & $-2,635$ & $-2,771$ & $-3,083$ & $-3,422$ \\
\hline Services, income, and transfers (net) & 96 & -24 & 33 & -374 \\
\hline Current transfers (net) & 1,400 & 1,273 & 1,384 & 1,201 \\
\hline Of which: workers' remittances & 1,223 & 1,228 & 1,230 & 1,137 \\
\hline Current account & 1,265 & 507 & 535 & 128 \\
\hline \multicolumn{5}{|l|}{ Capital and financial accounts, including errors } \\
\hline Overall balance & 1,368 & 761 & 752 & 583 \\
\hline
\end{tabular}


Table 2. Republic of Yemen: Selected Economic and Financial Indicators 2000-2003 (concluded)

\begin{tabular}{lrrrr}
\hline & 2000 & 2001 & 2002 & 2003 \\
\hline & & & & \\
Memorandum items: & 2,822 & 3,569 & 4,056 & 4,445 \\
$\quad$ Central bank own gross foreign reserves 3/ & 12.3 & 14.9 & 17.0 & 16.0 \\
$\quad$ In months of imports 3/ & 13.2 & 5.3 & 5.4 & 1.1 \\
Current account, including grants (in percent of GDP) & 6.0 & 6.3 & 4.6 & 3.9 \\
Debt service ratio (obligation basis), after rescheduling & 4,832 & 4,870 & 4,852 & 4,978 \\
Official external debt, after rescheduling 4/ & 167.2 & 133.0 & 138.1 & 141.2 \\
Terms of trade (1996=100) & 164.0 & 173.3 & 178.9 & 184.3 \\
Exchange rate (eop) (Yrls per U.S. dollar) & 164.6 & 182.0 & 189.4 & 178.8 \\
Real effective exchange rate (1995 = 100) & & & & \\
Nominal GDP at market prices & $1,539.4$ & $1,608.1$ & $1,753.5$ & $2,035.5$ \\
$\quad$ In billions of Yemeni rials & 9,561 & 9,533 & 9,985 & 11,211 \\
$\quad$ In millions of U.S. dollars & 546 & 528 & 537 & 585 \\
Per capita GDP (in U.S. dollars) & 17,507 & 18,046 & 18,601 & 19,173 \\
Population (in thousands) & & & & \\
\hline
\end{tabular}

Sources: Yemeni authorities; and Fund staff estimates.

$1 /$ Core CPI is defined as CPI excluding qat.

2/ Oil price is different from the WEO price because Yemeni oil is traded at a discount.

3/ Gross reserves minus commercial bank and pension fund foreign exchange deposits held with the cer bank. Imports are for the current year and exclude oil and gas sector imports.

4/ Public and publicly guaranteed debt including central bank foreign liabilities. Debt service ratio is measured as a percent of exports of goods and services. 
Table 3. Republic of Yemen: Sectoral Origin of Gross Domestic Product at Current Prices, 1998-2003

\begin{tabular}{|c|c|c|c|c|c|c|}
\hline & 1998 & 1999 & 2000 & 2001 & 2002 & 2003 \\
\hline & \multicolumn{6}{|c|}{ (In millions of Yemeni rials) } \\
\hline Agriculture and forestry & 113,180 & 130,404 & 142,679 & 160,759 & 169,988 & 186,579 \\
\hline Qat & 44,188 & 49,844 & 58,056 & 64,154 & 72,746 & 79,962 \\
\hline Fishing & 14,615 & 13,743 & 13,626 & 21,334 & 22,982 & 29,879 \\
\hline Mining and quarrying & 1,412 & 2,214 & 1,694 & 1,455 & 1,491 & 1,654 \\
\hline Oil and gas & 137,758 & 329,032 & 558,817 & 502,950 & 520,773 & 657,759 \\
\hline Manufacturing & 60,467 & 60,979 & 73,141 & 77,034 & 79,566 & 90,585 \\
\hline Oil refining & 5,345 & 6,216 & 6,651 & 7,818 & 9,490 & 10,256 \\
\hline Electricity, gas, water & 7,912 & 11,822 & 15,761 & 17,522 & 18,523 & 15,784 \\
\hline Construction & 53,157 & 62,927 & 64,342 & 64,606 & 79,321 & 86,597 \\
\hline Wholesale and retail trade & 84,594 & 110,357 & 145,037 & 172,391 & 194,003 & 245,689 \\
\hline Restaurants and hotels & 18,518 & 20,000 & 24,600 & 25,831 & 27,123 & 32,568 \\
\hline Maintenance & 8,627 & 9,463 & 10,384 & 11,396 & 12,504 & 13,722 \\
\hline Transportation, storage and communications & 109,780 & 131,629 & 156,149 & 176,838 & 194,895 & 229,874 \\
\hline Financial institutions & 30,643 & 41,899 & 43,362 & 55,296 & 52,228 & 56,069 \\
\hline Real estate and business services & 53,992 & 64,868 & 72,470 & 77,324 & 92,761 & 109,545 \\
\hline Community, social, and personal services & 9,054 & 11,008 & 13,101 & 14,089 & 17,969 & 19,854 \\
\hline Government services & 93,635 & 131,941 & 150,822 & 173,161 & 188,600 & 221,742 \\
\hline Household sector & 410 & 424 & 452 & 482 & 541 & 584 \\
\hline Private, non-profit services & 738 & 334 & 208 & 136 & 1,006 & 271 \\
\hline Import duties & 24,080 & 25,680 & 29,842 & 33,355 & 37,149 & 40,525 \\
\hline Less: Imputed bank services charges & $-27,865$ & $-41,990$ & $-41,808$ & $-49,866$ & $-40,154$ & $-47,854$ \\
\hline Total GDP & 844,240 & $1,172,794$ & $1,539,386$ & $1,608,065$ & $1,753,505$ & $2,035,491$ \\
\hline \multirow[t]{2}{*}{ Of which: non-oil GDP } & 706,482 & 843,762 & 980,569 & $1,105,115$ & $1,232,732$ & $1,434,543$ \\
\hline & \multicolumn{6}{|c|}{ (In percent of GDP) } \\
\hline Agriculture and forestry & 13.4 & 11.1 & 9.3 & 10.0 & 9.7 & 9.2 \\
\hline Qat & 5.2 & 4.3 & 3.8 & 4.0 & 4.1 & 3.9 \\
\hline Fishing & 1.7 & 1.2 & 0.9 & 1.3 & 1.3 & 1.5 \\
\hline Mining and quarrying & 0.2 & 0.2 & 0.1 & 0.1 & 0.1 & 0.1 \\
\hline Oil and gas & 16.3 & 28.1 & 36.3 & 31.3 & 29.7 & 32.3 \\
\hline Manufacturing & 7.2 & 5.2 & 4.8 & 4.8 & 4.5 & 4.5 \\
\hline Oil refining & 0.6 & 0.5 & 0.4 & 0.5 & 0.5 & 0.5 \\
\hline Electricity, gas, water & 0.9 & 1.0 & 1.0 & 1.1 & 1.1 & 0.8 \\
\hline Construction & 6.3 & 5.4 & 4.2 & 4.0 & 4.5 & 4.3 \\
\hline Wholesale and retail trade & 10.0 & 9.4 & 9.4 & 10.7 & 11.1 & 12.1 \\
\hline Restaurants and hotels & 2.2 & 1.7 & 1.6 & 1.6 & 1.5 & 1.6 \\
\hline Maintenance & 1.0 & 0.8 & 0.7 & 0.7 & 0.7 & 0.7 \\
\hline Transportation, storage, and communications & 13.0 & 11.2 & 10.1 & 11.0 & 11.1 & 11.3 \\
\hline Financial institutions & 3.6 & 3.6 & 2.8 & 3.4 & 3.0 & 2.8 \\
\hline Real estate and business services & 6.4 & 5.5 & 4.7 & 4.8 & 5.3 & 5.4 \\
\hline Community, social and personal services & 1.1 & 0.9 & 0.9 & 0.9 & 1.0 & 1.0 \\
\hline Government services & 11.1 & 11.3 & 9.8 & 10.8 & 10.8 & 10.9 \\
\hline Non-oil GDP & 83.7 & 71.9 & 63.7 & 68.7 & 70.3 & 70.5 \\
\hline
\end{tabular}

Sources: Central Statistical Organization. 
Table 4. Republic of Yemen: Sectoral Origin of Gross Domestic Product at Constant Prices, 1998-2003

\begin{tabular}{|c|c|c|c|c|c|c|}
\hline & 1998 & 1999 & 2000 & 2001 & 2002 & 2003 \\
\hline & \multicolumn{6}{|c|}{ (In millions of 1990 Yemeni rials) } \\
\hline Agriculture and forestry & 32,653 & 33,073 & 34,606 & 37,097 & 38,564 & 38,982 \\
\hline Qat & 13,142 & 13,471 & 13,884 & 14,296 & 14,708 & 16,706 \\
\hline Fishing & 1,385 & 1,398 & 1,453 & 1,492 & 1,542 & 1,625 \\
\hline Mining and quarrying & 364 & 368 & 382 & 356 & 352 & 364 \\
\hline Oil and gas & 30,326 & 32,719 & 35,806 & 36,280 & 36,430 & 35,784 \\
\hline Manufacturing & 14,438 & 14,518 & 14,821 & 15,165 & 15,906 & 16,542 \\
\hline Oil refining & 481 & 488 & 526 & 582 & 622 & 665 \\
\hline Electricity, gas, and water & 2,066 & 2,215 & 2,315 & 2,483 & 2,591 & 2,687 \\
\hline Construction & 4,494 & 4,548 & 4,721 & 4,832 & 5,049 & 5,321 \\
\hline Wholesale and retail trade & 12,512 & 12,110 & 12,964 & 14,452 & 15,075 & 15,755 \\
\hline Restaurants and hotels & 1,977 & 2,134 & 2,198 & 2,230 & 2,311 & 2,423 \\
\hline Maintenance & 1,478 & 1,531 & 1,588 & 1,646 & 1,708 & 1,773 \\
\hline Transportation, storage, and communications & 24,030 & 24,652 & 24,295 & 25,174 & 26,421 & 27,082 \\
\hline Financial institutions & 3,687 & 4,317 & 4,006 & 4,508 & 4,659 & 4,752 \\
\hline Real estate and business services & 10,181 & 10,827 & 11,597 & 12,383 & 13,168 & 14,076 \\
\hline Community, social, and personal services & 2,017 & 2,279 & 2,422 & 2,711 & 3,057 & 3,294 \\
\hline Government services & 47,852 & 50,673 & 52,660 & 54,700 & 56,888 & 58,768 \\
\hline Household sector & 310 & 311 & 314 & 316 & 320 & 322 \\
\hline Private, nonprofit services & 373 & 377 & 384 & 391 & 395 & 400 \\
\hline Import duties & 4,378 & 4,237 & 4,193 & 4,170 & 4,212 & 4,254 \\
\hline Less: Imputed bank services charges & $-3,353$ & $-4,354$ & $-3,866$ & $-3,912$ & $-3,532$ & $-3,569$ \\
\hline Total GDP at market prices & 204,791 & 211,892 & 221,269 & 231,352 & 240,446 & 248,005 \\
\hline \multicolumn{7}{|l|}{ Of which } \\
\hline \multirow[t]{2}{*}{ Non-oil GDP } & 174,465 & 179,173 & 185,463 & 195,072 & 204,016 & 212,221 \\
\hline & \multicolumn{6}{|c|}{ (Changes in percent) } \\
\hline Agriculture and forestry & 19.5 & 1.3 & 4.6 & 7.2 & 4.0 & 1.1 \\
\hline Qat & 2.6 & 2.5 & 3.1 & 3.0 & 2.9 & 13.6 \\
\hline Fishing & 9.3 & 0.9 & 3.9 & 2.7 & 3.4 & 5.4 \\
\hline Mining and quarrying & -9.7 & 1.1 & 3.8 & -6.8 & -1.1 & 3.4 \\
\hline Oil and gas & 2.7 & 7.9 & 9.4 & 1.3 & 0.4 & -1.8 \\
\hline Manufacturing & 5.5 & 0.6 & 2.1 & 2.3 & 4.9 & 4.0 \\
\hline Oil refining & -6.2 & 1.5 & 7.8 & 10.6 & 6.9 & 6.9 \\
\hline Electricity, gas, and water & 2.4 & 7.2 & 4.5 & 7.3 & 4.3 & 3.7 \\
\hline Construction & -10.0 & 1.2 & 3.8 & 2.4 & 4.5 & 5.4 \\
\hline Wholesale and retail trade & 9.4 & -3.2 & 7.1 & 11.5 & 4.3 & 4.5 \\
\hline Restaurants and hotels & 8.2 & 7.9 & 3.0 & 1.5 & 3.6 & 4.8 \\
\hline Maintenance & 3.7 & 3.6 & 3.7 & 3.7 & 3.8 & 3.8 \\
\hline Transportation, storage, and communications & 8.6 & 2.6 & -1.4 & 3.6 & 5.0 & 2.5 \\
\hline Financial institutions & 59.0 & 17.1 & -7.2 & 12.5 & 3.3 & 2.0 \\
\hline Real estate and business services & 6.4 & 6.3 & 7.1 & 6.8 & 6.3 & 6.9 \\
\hline Community, social, and personal services & 7.5 & 13.0 & 6.3 & 11.9 & 12.8 & 7.7 \\
\hline Government services & 0.9 & 5.9 & 3.9 & 3.9 & 4.0 & 3.3 \\
\hline GDP & 5.3 & 3.5 & 4.4 & 4.6 & 3.9 & 3.1 \\
\hline Non-oil GDP & 5.8 & 2.7 & 3.5 & 5.2 & 4.6 & 4.0 \\
\hline
\end{tabular}

Sources: Central Statistics Organization; and Central Bank of Yemen. 
Table 5. Republic of Yemen: Use of Resources at Current Prices, 1998-2003

\begin{tabular}{|c|c|c|c|c|c|c|}
\hline & 1998 & 1999 & 2000 & 2001 & 2002 & 2003 \\
\hline & \multicolumn{6}{|c|}{ (In millions of Yemeni rials) } \\
\hline Consumption & 816,316 & $1,035,429$ & $1,166,680$ & $1,303,138$ & $1,417,840$ & $1,610,544$ \\
\hline Public sector $1 /$ & 146,820 & 179,257 & 216,693 & 252,315 & 293,101 & 340,840 \\
\hline Private sector & 669,496 & 856,172 & 949,987 & $1,050,824$ & $1,124,739$ & $1,269,704$ \\
\hline Gross investment & 179,220 & 211,959 & 285,802 & 336,553 & 358,675 & 469,623 \\
\hline Gross fixed capital formation & 179,220 & 211,959 & 285,802 & 336,553 & 358,675 & 469,623 \\
\hline Public sector & 52,584 & 59,496 & 82,603 & 121,072 & 124,456 & 197,060 \\
\hline Private sector & 126,636 & 152,463 & 203,199 & 215,481 & 234,219 & 272,563 \\
\hline \multicolumn{7}{|l|}{ Of which } \\
\hline Oil companies expenditure & 16,850 & 23,246 & 58,911 & 62,741 & 79,436 & 82,420 \\
\hline Change in stocks & 0 & 0 & 0 & 0 & 0 & 0 \\
\hline Domestic absorption & 995,536 & $1,247,388$ & $1,452,482$ & $1,639,691$ & $1,776,515$ & $2,080,166$ \\
\hline Net exports of goods and nonfactor services & $-151,296$ & $-74,594$ & 86,904 & $-31,626$ & $-23,010$ & $-44,675$ \\
\hline GDP at market prices & 844,240 & $1,172,794$ & $1,539,386$ & $1,608,065$ & $1,753,505$ & $2,035,491$ \\
\hline Net factor income 2/ & $-42,360$ & $-98,108$ & $-108,513$ & $-97,659$ & $-126,090$ & $-150,193$ \\
\hline Gross national product (GNP) & 801,880 & $1,074,686$ & $1,430,873$ & $1,510,406$ & $1,627,415$ & $1,885,298$ \\
\hline Net current transfers $2 / 3 /$ & 170,340 & 204,675 & 225,320 & 214,747 & 243,001 & 218,025 \\
\hline Gross national disposable income (GNDI) & 972221 & 1279361 & 1656192 & 1725154 & 1870416 & 2103322 \\
\hline Gross domestic saving & 27,924 & 137,365 & 372,706 & 304,927 & 335,665 & 424,948 \\
\hline Gross national savings from disposable income & 155,904 & 243,932 & 489,512 & 422,015 & 452,576 & 492,779 \\
\hline Public sector national savings & $-1,537$ & 56,877 & 205,752 & 162,390 & 131,229 & 107,675 \\
\hline \multirow[t]{2}{*}{ Private sector national savings } & 157,441 & 187,055 & 283,760 & 259,626 & 321,347 & 385,104 \\
\hline & \multicolumn{6}{|c|}{ (In percent of GDP) } \\
\hline Consumption & 96.7 & 88.3 & 75.8 & 81.0 & 80.9 & 79.1 \\
\hline Public sector $1 /$ & 17.4 & 15.3 & 14.1 & 15.7 & 16.7 & 16.7 \\
\hline Private sector & 79.3 & 73.0 & 61.7 & 65.3 & 64.1 & 62.4 \\
\hline Gross investment & 21.2 & 18.1 & 18.6 & 20.9 & 20.5 & 23.1 \\
\hline Gross fixed capital formation & 21.2 & 18.1 & 18.6 & 20.9 & 20.5 & 23.1 \\
\hline Public sector & 6.2 & 5.1 & 5.4 & 7.5 & 7.1 & 9.7 \\
\hline Private sector & 15.0 & 13.0 & 13.2 & 13.4 & 13.4 & 13.4 \\
\hline \multicolumn{7}{|l|}{ Of which } \\
\hline Oil companies expenditures & 2.0 & 2.0 & 3.8 & 3.9 & 4.5 & 4.0 \\
\hline Change in stocks & 0.0 & 0.0 & 0.0 & 0.0 & 0.0 & 0.0 \\
\hline Domestic absorption & 117.9 & 106.4 & 94.4 & 102.0 & 101.3 & 102.2 \\
\hline Net exports of goods and nonfactor services & -17.9 & -6.4 & 5.6 & -2.0 & -1.3 & -2.2 \\
\hline Net factor income 2/ & -5.0 & -8.4 & -7.0 & -6.1 & -7.2 & -7.4 \\
\hline Gross national product & 95.0 & 91.6 & 93.0 & 93.9 & 92.8 & 92.6 \\
\hline Net current transfers $2 / 3 /$ & 20.2 & 17.5 & 14.6 & 13.4 & 13.9 & 10.7 \\
\hline Gross national disposable income & 115.2 & 109.1 & 107.6 & 107.3 & 106.7 & 103.3 \\
\hline Gross domestic saving & 3.3 & 11.7 & 24.2 & 19.0 & 19.1 & 20.9 \\
\hline Gross national savings from disposable income & 18.5 & 20.8 & 31.8 & 26.2 & 25.8 & 24.2 \\
\hline Public sector national savings & -0.2 & 4.8 & 13.4 & 10.1 & 7.5 & 5.3 \\
\hline Private sector national savings & 18.6 & 15.9 & 18.4 & 16.1 & 18.3 & 18.9 \\
\hline
\end{tabular}

Sources: Yemeni authorities; and Fund staff estimates.

1 / Uses data from the fiscal accounts.

2/ Uses balance of payments data.

3 / Includes workers' remittances. 


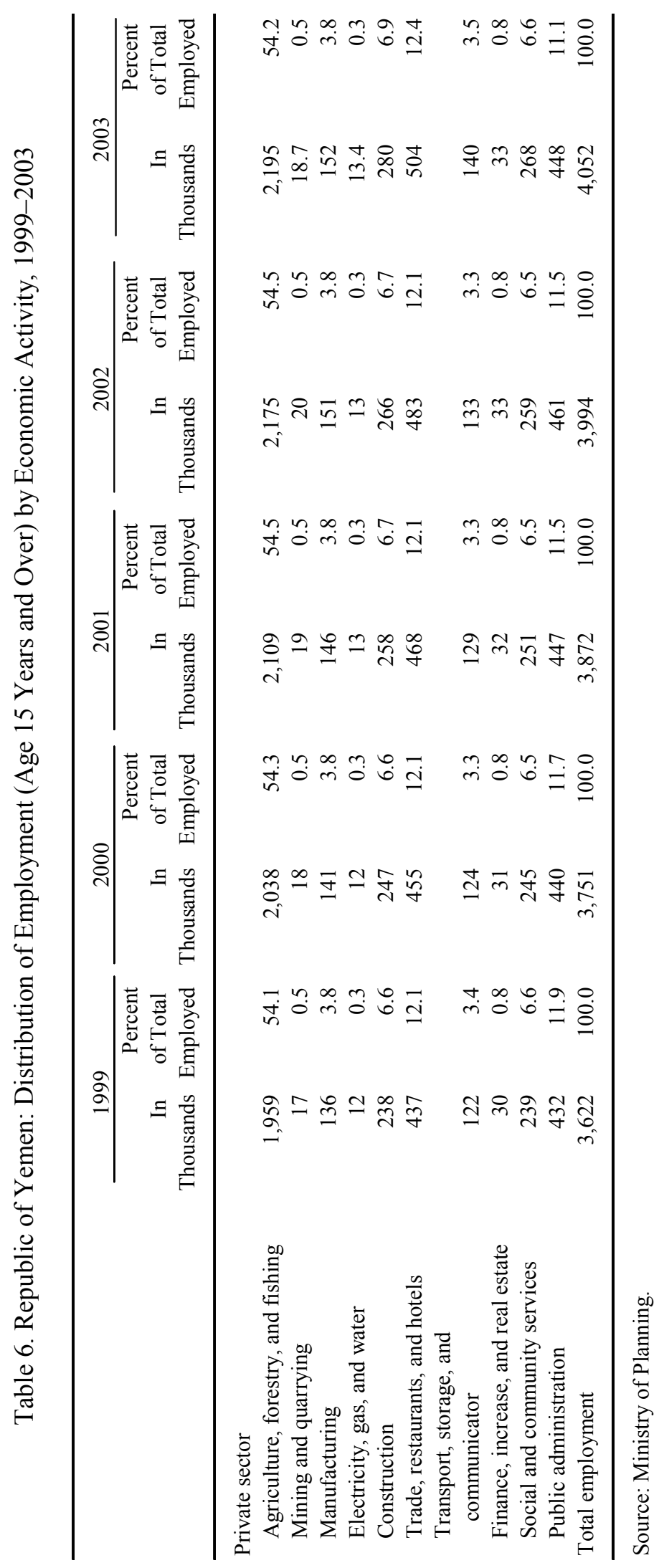




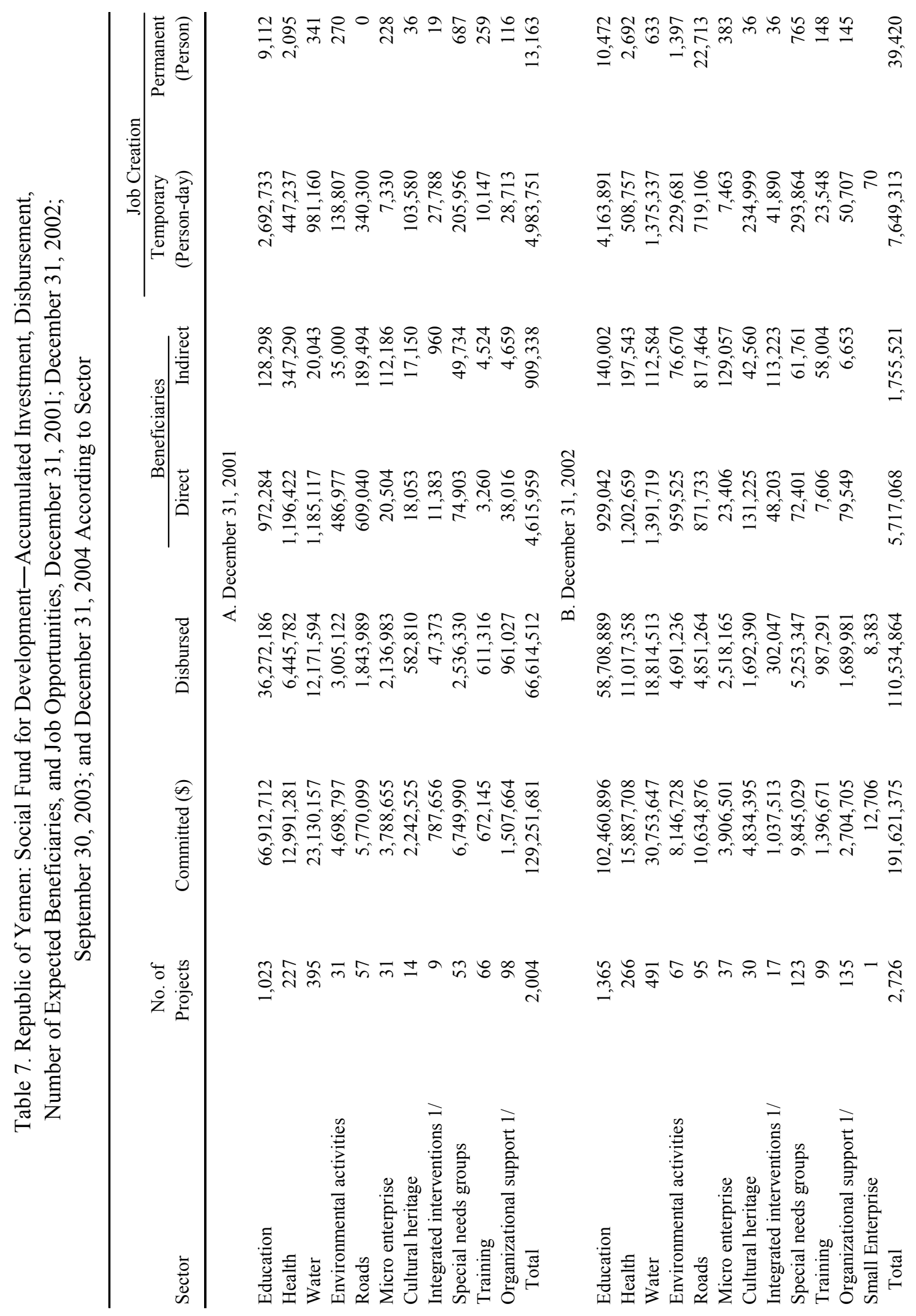




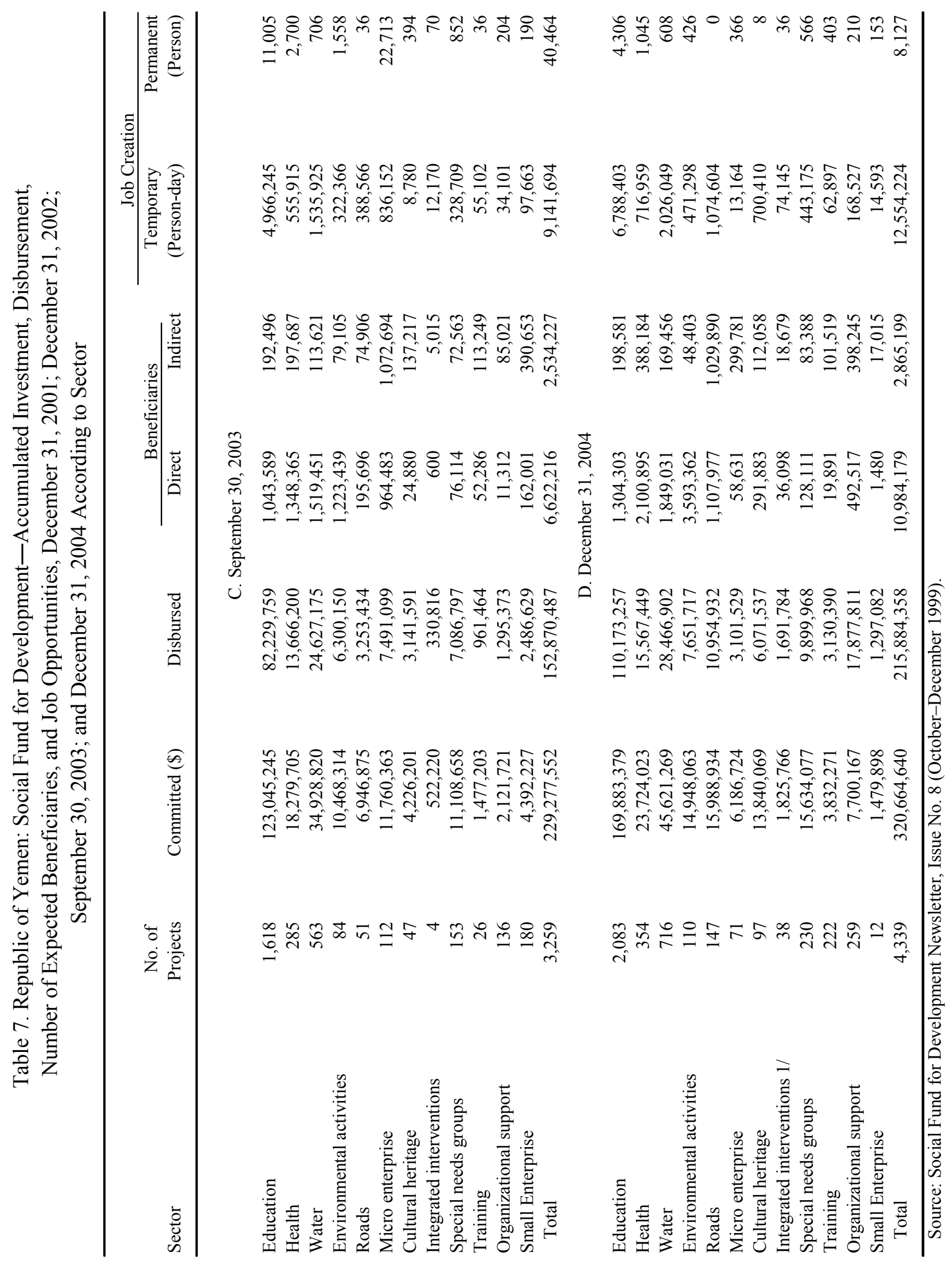


Table 8. Republic of Yemen: Social Fund for Development—-Total Investment and Disbursement up to September 30, 2004

According to Governorates

\begin{tabular}{lcccc}
\hline Governorate & & & & Percentage of \\
No. of Projects & Committed $(\$)$ & Disbursed $(\$)$ & 77 \\
\hline Abian & 98 & $6,970,359$ & $5,391,834$ & 81 \\
Aden & 130 & $11,577,927$ & $9,385,559$ & 62 \\
Al-Dhaleea & 65 & $5,261,639$ & $3,259,702$ & 48 \\
Al-Jawf & 73 & $5,180,784$ & $2,490,208$ & 78 \\
Amran & 255 & $17,770,147$ & $13,863,417$ & 77 \\
Baidhaa & 108 & $9,005,681$ & $6,930,278$ & 72 \\
Dhamar & 302 & $19,204,392$ & $13,822,404$ & 69 \\
Hadhramout & 272 & $17,622,355$ & $12,144,434$ & 71 \\
Hajjah & 256 & $18,644,012$ & $13,164,906$ & 68 \\
Hodiedah & 366 & $29,785,815$ & $20,293,827$ & 52 \\
Ibb & 337 & $25,906,208$ & $13,525,692$ & 65 \\
Lahj & 132 & $9,139,157$ & $5,959,177$ & 37 \\
Maareb & 61 & $5,542,254$ & $2,027,580$ & 95 \\
Maharah & 40 & $2,380,811$ & $2,250,658$ & 73 \\
Mahweet & 134 & $11,149,807$ & $8,128,623$ & 73 \\
Saadah & 103 & $7,372,423$ & $5,346,707$ & 66 \\
Sana'a & 310 & $19,833,899$ & $13,099,724$ & 68 \\
Sana'a capital & 388 & $35,491,480$ & $24,298,934$ & 66 \\
Shabwah & 121 & $7,134,936$ & $4,741,746$ & 61 \\
Taiz & 442 & $31,538,195$ & $19,342,901$ & 67 \\
Other governorates & 212 & $8,736,082$ & $5,829,392$ & 67 \\
Total & & & & \\
& 4,205 & $305,248,363$ & $205,297,703$ & \\
\hline & & & & \\
\hline
\end{tabular}

Source: Social Fund for Development. 
Table 9. Republic of Yemen: Distribution of Labor Force by Employment Status, Age Group (+10 Years), and Gender, 1994, 1999, and 2002

(In percent)

\begin{tabular}{|c|c|c|c|c|c|c|}
\hline \multirow[b]{2}{*}{ Age Group } & \multicolumn{2}{|c|}{ Unemployed } & \multirow[b]{2}{*}{ Total } & \multicolumn{2}{|c|}{ Total Labor Force } & \multirow[b]{2}{*}{ Total } \\
\hline & Male & Female & & Male & Female & \\
\hline \multicolumn{7}{|c|}{ (1994) } \\
\hline $10-14$ & 11.3 & 39.5 & 14.7 & 3.4 & 14.7 & 5.7 \\
\hline $15-19$ & 22.9 & 31.5 & 23.9 & 7.7 & 13.7 & 8.9 \\
\hline $20-24$ & 21.5 & 14.7 & 20.7 & 11.9 & 11.1 & 11.8 \\
\hline $25-29$ & 13.0 & 6.2 & 12.1 & 14.0 & 12.9 & 13.8 \\
\hline $30-34$ & 7.5 & 2.5 & 6.9 & 13.2 & 11.9 & 12.9 \\
\hline $35-39$ & 6.1 & 1.5 & 5.6 & 13.0 & 11.6 & 12.7 \\
\hline $40-44$ & 4.3 & 1.0 & 3.9 & 9.6 & 8.4 & 9.4 \\
\hline $45-49$ & 3.4 & 0.6 & 3.1 & 7.6 & 6.1 & 7.3 \\
\hline $50-54$ & 3.1 & 0.7 & 2.8 & 6.6 & 2.2 & 5.7 \\
\hline $55-59$ & 1.9 & 0.4 & 1.7 & 3.5 & 2.4 & 3.3 \\
\hline $60-64$ & 2.1 & 0.5 & 1.9 & 4.3 & 2.6 & 4.0 \\
\hline $65+$ & 2.8 & 0.9 & 2.6 & 5.1 & 2.4 & 4.5 \\
\hline Unspecified & 0.0 & 0.1 & 0.0 & 0.0 & 0.0 & 0.0 \\
\hline Total & 100.0 & 100.0 & 100.0 & 100.0 & 100.0 & 100.0 \\
\hline Total (In thousands) & \multicolumn{6}{|c|}{ (1999) } \\
\hline $15-19$ & 23.0 & 29.0 & 24.0 & 13.0 & 19.0 & 15.0 \\
\hline $20-24$ & 24.0 & 27.0 & 25.0 & 15.0 & 15.0 & 15.0 \\
\hline $25-29$ & 16.0 & 20.0 & 17.0 & 15.0 & 14.0 & 15.0 \\
\hline $30-34$ & 9.0 & 10.0 & 9.0 & 11.0 & 11.0 & 11.0 \\
\hline $35-39$ & 8.0 & 5.0 & 8.0 & 12.0 & 12.0 & 12.0 \\
\hline $40-44$ & 7.0 & 4.0 & 7.0 & 9.0 & 11.0 & 10.0 \\
\hline $45-49$ & 5.0 & 2.0 & 4.0 & 8.0 & 6.0 & 7.0 \\
\hline $50-54$ & 3.0 & 2.0 & 3.0 & 6.0 & 5.0 & 6.0 \\
\hline $55-59$ & 2.0 & 1.0 & 2.0 & 3.0 & 3.0 & 3.0 \\
\hline $60-64$ & 1.0 & 1.0 & 1.0 & 4.0 & 2.0 & 3.0 \\
\hline $65+$ & 1.0 & 0.0 & 1.0 & 3.0 & 2.0 & 3.0 \\
\hline \multicolumn{7}{|l|}{ Unspecified } \\
\hline Total & 100.0 & 100.0 & 100.0 & 100.0 & 100.0 & 100.0 \\
\hline Total (In thousands) & 390 & 79 & 469 & 3,121 & 969 & 4,091 \\
\hline \multicolumn{7}{|c|}{ (2002) } \\
\hline $15-19$ & 23.0 & 29.0 & 24.0 & 13.0 & 19.0 & 15.0 \\
\hline $20-24$ & 24.0 & 27.0 & 25.0 & 15.0 & 15.0 & 15.0 \\
\hline $25-29$ & 16.0 & 20.0 & 17.0 & 15.0 & 14.0 & 15.0 \\
\hline $30-34$ & 9.0 & 10.0 & 9.0 & 11.0 & 11.0 & 11.0 \\
\hline $35-39$ & 8.0 & 5.0 & 8.0 & 12.0 & 12.0 & 12.0 \\
\hline $40-44$ & 7.0 & 4.0 & 7.0 & 9.0 & 11.0 & 10.0 \\
\hline $45-49$ & 5.0 & 2.0 & 4.0 & 8.0 & 6.0 & 7.0 \\
\hline $50-54$ & 3.0 & 2.0 & 3.0 & 6.0 & 5.0 & 6.0 \\
\hline $55-59$ & 2.0 & 1.0 & 2.0 & 3.0 & 3.0 & 3.0 \\
\hline $60-64$ & 1.0 & 1.0 & 1.0 & 4.0 & 2.0 & 3.0 \\
\hline & 1.0 & 0.0 & 1.0 & 3.0 & 2.0 & 3.0 \\
\hline \multicolumn{7}{|l|}{ Unspecified } \\
\hline Total & 100.0 & 100.0 & 100.0 & 100.0 & 100.0 & 100.0 \\
\hline Total (In thousands) & 390 & 79 & 469 & 3,121 & 969 & 4,091 \\
\hline
\end{tabular}

Sources: Central Statistics Organization; General Population Census 1994 and 1999. 


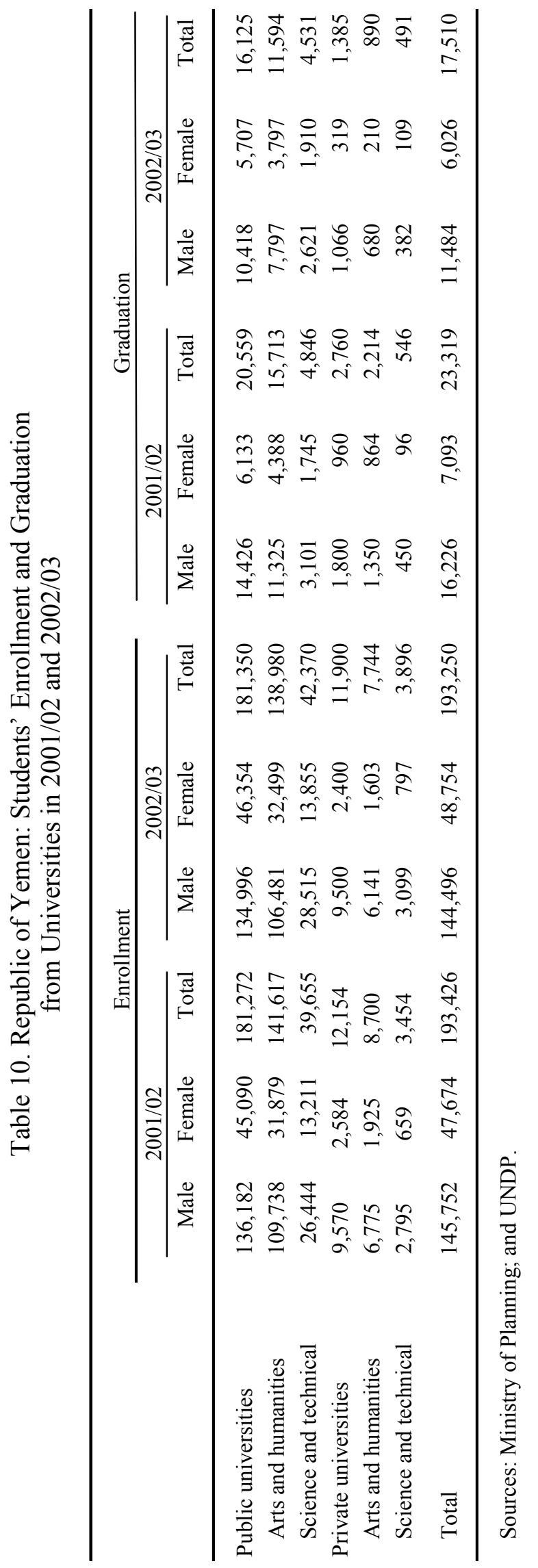


Table 11. Republic of Yemen: Crude Oil Summary, 1998-2003

(In thousands of barrels per day)

\begin{tabular}{|c|c|c|c|c|c|c|}
\hline & 1998 & 1999 & 2000 & 2001 & 2002 & 2003 \\
\hline Total net output $1 /$ & 367 & 389 & 434 & 434 & 433 & 425 \\
\hline Marib & 129 & 116 & 123 & 114 & 107 & 99 \\
\hline Masila & 201 & 207 & 215 & 228 & 227 & 225 \\
\hline Jannah & 19 & 48 & 65 & 57 & 42 & 42 \\
\hline East Shabwa & 16 & 17 & 29 & 27 & 25 & 23 \\
\hline Ayad & 1 & 1 & 1 & 1 & 0 & 0 \\
\hline East Sarr & $\ldots$ & $\ldots$ & $\ldots$ & $\ldots$ & 20 & 19 \\
\hline Howarime & $\ldots$ & $\ldots$ & $\ldots$ & $\ldots$ & 11 & 17 \\
\hline Companies' exports & 177 & 158 & 156 & 158 & 175 & 164 \\
\hline Marib & 52 & 47 & 40 & 43 & 42 & 37 \\
\hline Masila & 104 & 77 & 69 & 76 & 85 & 84 \\
\hline Jannah 2/ & 8 & 20 & 27 & 18 & 15 & 14 \\
\hline East Shabwa & 13 & 13 & 19 & 15 & 15 & 12 \\
\hline Ayad & 1 & 1 & 1 & 0 & 0 & 0 \\
\hline East Sarr & $\ldots$ & $\ldots$ & $\ldots$ & $\ldots$ & 11 & 8 \\
\hline Howarime & $\ldots$ & $\ldots$ & $\ldots$ & $\ldots$ & 8 & 9 \\
\hline Companies' net output share & 100 & 112 & 132 & 125 & 124 & 99 \\
\hline Marib & 61 & 56 & 66 & 58 & 54 & 26 \\
\hline Masila & 35 & 47 & 52 & 54 & 50 & 50 \\
\hline Jannah 2/ & 3 & 7 & 9 & 7 & 9 & 10 \\
\hline East Shabwa & 1 & 2 & 4 & 5 & 4 & 4 \\
\hline Ayad & 0.20 & 0.10 & 0.10 & 0.00 & 0 & 0 \\
\hline East Sarr & $\ldots$ & $\ldots$ & $\ldots$ & $\ldots$ & 4 & 5 \\
\hline Howarime & $\ldots$ & $\ldots$ & $\ldots$ & $\ldots$ & 2 & 4 \\
\hline Cost recovery & 107 & 75 & 57 & 62 & 79 & 65 \\
\hline Marib & 21 & 18 & 7 & 14 & 15 & 11 \\
\hline Masila & 69 & 31 & 17 & 22 & 35 & 34 \\
\hline Jannah & 5 & 13 & 18 & 11 & 6 & 4 \\
\hline East Shabwa & 11 & 12 & 15 & 10 & 10 & 8 \\
\hline Ayad & 0.83 & 0.64 & 0.49 & 0.40 & 0 & 0 \\
\hline East Sarr & $\ldots$ & $\ldots$ & $\ldots$ & $\ldots$ & 7 & 3 \\
\hline Howarime & $\ldots$ & $\ldots$ & $\ldots$ & $\ldots$ & 7 & 5 \\
\hline Income tax & 30 & 28 & 33 & 29 & 27 & 26 \\
\hline Marib & 30 & 28 & 33 & 29 & 27 & 26 \\
\hline Masila & 0 & 0 & 0 & 0 & 0 & 0 \\
\hline Jannah & 0 & 0 & 0 & 0 & 0 & 0 \\
\hline East Shabwa & 0 & 0 & 0 & 0 & 0 & 0 \\
\hline Ayad & 0 & 0 & 0 & 0 & 0 & 0 \\
\hline East Sarr & $\ldots$ & $\ldots$ & $\ldots$ & $\ldots$ & 0 & 0 \\
\hline Howarime & $\ldots$ & $\ldots$ & $\ldots$ & $\ldots$ & 0 & 0 \\
\hline Government exports & 112 & 146 & 188 & 187 & 173 & 179 \\
\hline Government sales to refineries & 77 & 85 & 89 & 90 & 85 & 82 \\
\hline Average oil export price (US\$/barrel) & 11.71 & 19.74 & 25.88 & 23.02 & 24.6 & 28 \\
\hline Export revenue (In millions of US\$ dollars & $1,228.7$ & $2,131.2$ & $3,422.7$ & $2,894.0$ & $3,146.6$ & $3,452.7$ \\
\hline Companies & 758.0 & $1,138.2$ & $1,479.2$ & $1,325.8$ & $1,546.6$ & $1,624.3$ \\
\hline Government & 470.7 & 993.0 & $1,943.5$ & $1,568.2$ & $1,600.0$ & $1,828.4$ \\
\hline
\end{tabular}

Source: Ministry of Oil and Mineral Resources.

$1 /$ Net of miscellaneous oil field uses.

2/ Including YICOM share. 
Table 12. Republic of Yemen: Domestic Consumption of Petroleum Products, 1998-2003

\begin{tabular}{lrrrrrr}
\hline & 1998 & 1999 & 2000 & 2001 & 2002 & 2003 \\
\hline & & \multicolumn{7}{c}{ (In millions of liters) } \\
Total petroleum products & 4,043 & 3,911 & $\ldots$ & $\ldots$ & $\ldots$ & 5,880 \\
Gasoline & 1,322 & 1,107 & 1,134 & 1,364 & 1,394 & 1,472 \\
Diesel & 1,215 & 1,302 & 1,533 & 1,922 & 2,126 & 2,527 \\
Kerosene & 150 & 140 & 289 & 279 & 420 & 132 \\
Aviation fuel & 99 & 111 & $\ldots$ & $\ldots$ & $\ldots$ & 133 \\
Fuel oil & 938 & 898 & 998 & 1,122 & 973 & 992 \\
LPG & 318 & 353 & 464 & $\ldots$ & 592 & 624 \\
& & & $($ percent change) & & & \\
Total petroleum products & -2.7 & -3.3 & $\ldots$ & $\ldots$ & $\ldots$ & $\ldots$ \\
Gasoline & -6.9 & -16.2 & 2.4 & 20.3 & 2.2 & 5.6 \\
Diesel & -4.6 & 7.1 & 17.7 & 25.4 & 10.6 & 18.9 \\
Kerosene & -8.0 & -6.7 & 106.6 & -3.5 & 50.5 & -68.6 \\
Aviation fuel & 6.2 & 12.6 & $\ldots$ & $\ldots$ & $\ldots$ & $\ldots$ \\
Fuel oil & 3.2 & -4.3 & 11.2 & 12.4 & -13.3 & 2.0 \\
LPG & 7.2 & 10.8 & 31.6 & $\ldots$ & 16.9 & 5.4 \\
\hline
\end{tabular}

Source: Ministry of Finance. 
Table 13. Republic of Yemen: Output of Industrial Products, 1998-2003

\begin{tabular}{|c|c|c|c|c|c|c|}
\hline & 1998 & 1999 & 2000 & 2001 & 2002 & 2003 \\
\hline Electricity (million kwh) & 2,912 & 3,136 & 3,413 & 3,643 & 3,769 & 4,094 \\
\hline \multicolumn{7}{|l|}{ Extractive industries } \\
\hline Salt (thousand tons) & 144 & 149 & 95 & 113 & 125 & 116 \\
\hline Quarried stone (thousand cubic meters) & 2,371 & 2,476 & 2,480 & 2,243 & 2,288 & 2,333 \\
\hline Gypsum (thousand tons) & 37 & 40 & 42 & 44 & 41 & 42 \\
\hline Crude oil (millions of barrels) & $\ldots$ & 135 & 159 & 160 & 160 & 157 \\
\hline \multicolumn{7}{|l|}{ Food, beverage, and tobacco industries } \\
\hline Bread (thousand tons) & 170 & 179 & $\ldots$ & $\ldots$ & $\ldots$ & $\ldots$ \\
\hline Biscuits and confectioneries (thousand tons) & 44 & 45 & 46 & 51 & 52 & 54 \\
\hline Ghee and edible oils (thousand tons) & 134 & 133 & 103 & 102 & 110 & 114 \\
\hline Soft drinks (million liters) & 69 & 80 & 81 & 94 & 103 & 105 \\
\hline Mineral water (thousand cubic meters) & 68 & 92 & 98 & 133 & 140 & 143 \\
\hline Ice (thousand tons) & 61 & 61 & 62 & 63 & $\ldots$ & $\ldots$ \\
\hline Milk and products (million liters) & 41 & 48 & 63 & 64 & 70 & 71 \\
\hline Ice cream (tons) & 1,715 & 1,749 & $\ldots$ & $\ldots$ & $\ldots$ & $\ldots$ \\
\hline Cigarettes (million packs) & 302 & 293 & 238 & 301 & 289 & 298 \\
\hline Canned fish(thousand cans) & $\ldots$ & 7,162 & 14,072 & 28,120 & 34,896 & 33,849 \\
\hline \multicolumn{7}{|l|}{ Metallic industries } \\
\hline Household utensils (tons) & 1,077 & 1,098 & 1,102 & 1,127 & 879 & 896 \\
\hline Barrels (thousands) & 723 & 730 & $\ldots$ & $\ldots$ & $\ldots$ & $\ldots$ \\
\hline Doors and windows (thousand square meters) & 744 & 747 & 752 & 908 & 854 & 871 \\
\hline \multicolumn{7}{|l|}{ Nonmetallic industries } \\
\hline Cement (thousand tons) & 1,195 & 1,454 & 1,406 & 1,449 & 1,561 & 1,541 \\
\hline Red bricks (thousands) & 14,897 & 15,120 & $\ldots$ & & $\ldots$ & $\ldots$ \\
\hline Cement bricks (millions) & 94 & 97 & 98 & 102 & 104 & 106 \\
\hline Tiles (millions) & 76 & 78 & 79 & 81 & 83 & 85 \\
\hline Marble (thousand square meters) & 93 & 97 & 99 & 101 & 99 & 100 \\
\hline \multicolumn{7}{|l|}{ Chemical industries } \\
\hline Plastic footwear (millions of pairs) & 6 & 5 & 6 & 6 & 6 & 7 \\
\hline Household utensils (tons) & 4,081 & 4,111 & 4,143 & 4,171 & 4,129 & 4,191 \\
\hline Water tubes (tons) & 3,653 & 3,683 & 3,947 & 5,304 & 5,039 & 5,140 \\
\hline Plastic sheets (tons) & 10,181 & 10,211 & 10,321 & 10,733 & 12,128 & 12,249 \\
\hline Paint (thousand tons) & 7,513 & 7,573 & 7,205 & 7,479 & 7,554 & 7,667 \\
\hline Soap (thousand tons) & 61 & 51 & 58 & 43 & 50 & 51 \\
\hline \multicolumn{7}{|l|}{ Paper and printing } \\
\hline Paper tissues (tons) & 1,390 & 1,436 & 1,498 & 1,281 & 1,307 & 1,327 \\
\hline School books (millions of books) & 22 & 26 & 33 & 73 & 75 & $\ldots$ \\
\hline Cartons (thousand tons) & 23 & 27 & 29 & 29 & $\ldots$ & $\ldots$ \\
\hline
\end{tabular}

Source: Central Statistics Organization. 
Table 14. Republic of Yemen: Production, Area, and Yield of Major Crops, 1998-2003 1/

(Production in thousand metric tons; area in thousand hectares; and yield in tons per hectare)

\begin{tabular}{|c|c|c|c|c|c|c|}
\hline & 1998 & 1999 & 2000 & 2001 & 2002 & 2003 \\
\hline \multicolumn{7}{|l|}{ Cereals (total) } \\
\hline Production & 833.3 & 693.7 & 672.2 & 700.1 & 559.8 & 477.4 \\
\hline Area & 770.4 & 624.3 & 619.6 & 657.9 & 593.1 & 581.5 \\
\hline \multicolumn{7}{|c|}{ Sorghum and millet } \\
\hline Production & 609.8 & 511.3 & 487.9 & 501.4 & 388.3 & 286.1 \\
\hline Area & 609.7 & 501.4 & 495.2 & 522.2 & 463.6 & 378.0 \\
\hline Yield & 1.0 & 1.0 & 1.0 & 1.0 & 0.8 & 0.7 \\
\hline \multicolumn{7}{|l|}{ Wheat } \\
\hline Production & 167.4 & 139.6 & 141.9 & 152.7 & 131.7 & 103.8 \\
\hline Area & 111.3 & 86.1 & 87.3 & 94.4 & 89.4 & 86.5 \\
\hline Yield & 1.5 & 1.6 & 1.6 & 1.6 & 1.5 & 1.2 \\
\hline \multicolumn{7}{|l|}{ Barley } \\
\hline Production & 56.2 & 42.8 & 42.4 & 46.0 & 39.8 & 27.9 \\
\hline Area & 49.4 & 36.8 & 37.0 & 41.3 & 40.1 & 37.7 \\
\hline Yield & 1.1 & 1.2 & 1.1 & 1.1 & 1.0 & 0.7 \\
\hline \multicolumn{7}{|l|}{ Legumes } \\
\hline Production & 78.0 & 64.2 & 63.1 & 64.0 & 60.5 & 59.5 \\
\hline Area & 62.5 & 51.1 & 51.5 & 52.7 & 49.6 & 49.2 \\
\hline Yield & 1.2 & 1.3 & 1.2 & 1.2 & 1.2 & 1.2 \\
\hline \multicolumn{7}{|l|}{ Potatoes } \\
\hline Production & 196.5 & 211.1 & 210.4 & 208.6 & 210.8 & 213.3 \\
\hline Area & 15.6 & 16.7 & 17.0 & 17.3 & 17.6 & 17.8 \\
\hline Yield & 12.6 & 12.6 & 12.4 & 12.1 & 12.0 & 12.0 \\
\hline \multicolumn{7}{|l|}{ Vegetables } \\
\hline Production & 550.7 & 546.7 & 564.5 & 594.1 & 608.2 & 620.0 \\
\hline Area & 46.2 & 45.8 & 50.0 & 50.1 & 52.0 & 53.4 \\
\hline Yield & 11.9 & 11.9 & 11.3 & 11.9 & 11.7 & 11.6 \\
\hline \multicolumn{7}{|c|}{ Grapes, dates, and other fruits } \\
\hline Production & 591.1 & 626.3 & 654.9 & 703.4 & 719.8 & 736.2 \\
\hline Area & 84.1 & 88.1 & 91.2 & 95.2 & 97.1 & 98.3 \\
\hline \multicolumn{7}{|l|}{ Grapes } \\
\hline Production & 154.9 & 155.7 & 155.9 & 162.7 & 164.6 & 168.8 \\
\hline Area & 22.1 & 22.7 & 22.6 & 22.7 & 22.8 & 22.9 \\
\hline Yield & 7.0 & 6.9 & 6.9 & 7.2 & 7.2 & 7.4 \\
\hline \multicolumn{7}{|l|}{ Dates } \\
\hline Production & 26.9 & 28.5 & 29.8 & 31.6 & 32.4 & 33.3 \\
\hline Area & 20.6 & 22.2 & 22.8 & 23.1 & 23.4 & 23.6 \\
\hline Yield & 1.3 & 1.3 & 1.3 & 1.4 & 1.4 & 1.4 \\
\hline
\end{tabular}


Table 14. Republic of Yemen: Production, Area, and Yield of Major Crops, 1998-2003 1/ (concluded)

(Production in thousand metric tons; area in thousand hectares; and yield in tons per hectare)

\begin{tabular}{lrrrrrr}
\hline & 1998 & 1999 & 2000 & 2001 & 2002 & 2003 \\
& & & & & & \\
& & & & & & \\
Other fruits & & & & & & \\
$\quad$ Production & 409.3 & 442.1 & 469.2 & 509.1 & 522.8 & 534.1 \\
$\quad$ Area & 41.3 & 43.5 & 45.7 & 49.4 & 50.9 & 51.8 \\
$\quad$ Yield & 9.0 & 9.1 & 8.9 & 8.7 & 10.3 & 10.3 \\
Coffee & & & & & & \\
$\quad$ Production & 11.3 & 11.8 & 11.4 & 11.9 & 11.5 & 11.6 \\
Area & 32.0 & 32.8 & 33.4 & 33.6 & 33.5 & 33.7 \\
Yield & 0.4 & 0.4 & 0.3 & 0.4 & 0.3 & 0.3 \\
Tobacco & & & & & & \\
Production & 11.5 & 11.1 & 11.6 & 12.1 & 11.5 & 11.9 \\
Area & 5.4 & 5.2 & 5.3 & 5.5 & 5.4 & 5.5 \\
Yield & 2.1 & 2.1 & 2.2 & 2.2 & 2.2 & 2.2 \\
Sesame & & & & & & \\
Production & 17.5 & 17.8 & 18.3 & 19.4 & 18.6 & 18.7 \\
Area & 30.3 & 31.0 & 32.0 & 33.1 & 32.4 & 32.5 \\
Yield & 0.6 & 0.6 & 0.6 & 0.6 & 0.6 & 0.6 \\
Alfalfa & & & & & & \\
Production & 222.9 & 234.4 & 237.4 & 240.7 & 238.7 & 241.6 \\
Area & 24.5 & 25.8 & 26.1 & 26.8 & 26.6 & 26.7 \\
Yield & 9.1 & 9.1 & 9.1 & 9.0 & 9.0 & 9.0 \\
\hline
\end{tabular}

Sources: Ministry of Agriculture; and Central Statistics Organization.

1/ Data for 2003 is preliminary. 
Table 15. Republic of Yemen: Noncrop Primary Production, 1998-2003

(In thousands of metric tons)

\begin{tabular}{lrrrrrr}
\hline & 1998 & 1999 & 2000 & 2001 & 2002 & 2003 \\
\hline Total meat and milk & 274.9 & 286.8 & 298.2 & 324.0 & 335.4 & 343.3 \\
$\quad$ Meat & 45.3 & 47.1 & 51.7 & 56.2 & 59.3 & 59.8 \\
Poultry & 61.4 & 63.1 & 66.7 & 78.3 & 83.5 & 87.3 \\
Milk & 168.2 & 176.6 & 179.8 & 189.5 & 192.6 & 196.2 \\
Eggs (in millions) & 600.0 & 603.0 & 604.0 & 611.0 & 613.0 & 615.0 \\
Total fish catch & 127.6 & 123.6 & 134.7 & 142.2 & 179.6 & 228.1 \\
Surface water fish & 104.9 & 110.1 & 120.0 & 122.5 & 151.9 & 202.8 \\
$\quad$ Deep water fish & 12.1 & 7.9 & 8.5 & 7.8 & 19.6 & 10.9 \\
$\quad$ Other aquatic catch & 10.6 & 5.6 & 6.2 & 11.9 & 8.1 & 14.4 \\
\hline
\end{tabular}

Sources: Central Statistics Organization; and Ministry of Agriculture. 
Table 16. Republic of Yemen: Consumer Price Index, 1998-2003 1/

\begin{tabular}{lrrrrrr}
\hline & 1998 & 1999 & 2000 & 2001 & 2002 & 2003 \\
\hline & \multicolumn{7}{c}{ (November 1999=100) } \\
Overall index & 92.03 & 100.00 & 104.59 & 117.05 & 131.37 & 145.60 \\
Food stuffs & 90.60 & 100.00 & 104.87 & 118.89 & 127.62 & 148.87 \\
Housing & 90.75 & 100.00 & 103.17 & 108.62 & 114.91 & 123.07 \\
Clothing & 115.96 & 100.00 & 104.35 & 108.40 & 116.92 & 120.82 \\
Others & 94.50 & 100.00 & 103.30 & 113.29 & 119.83 & 124.37 \\
& & & & & \\
Overall index & 6.0 & 8.7 & 4.6 & 11.9 & 12.2 & 10.8 \\
Food stuffs & 5.5 & 10.4 & 4.9 & 13.4 & 7.34 & 16.65 \\
Housing & 7.1 & 10.2 & 3.2 & 5.3 & 5.70 & 7.10 \\
Clothing & 10.2 & -13.8 & 4.3 & 3.9 & 7.0 & 3.34 \\
Others & 5.6 & 5.8 & 3.3 & 9.7 & 5.7 & 3.79 \\
\hline
\end{tabular}

Sources: Central Statistics Organization; and Fund staff estimates.

1/ The CPI for 1998-99 is based on consumption shares from the 1992 Household Budget Survey and consists of weighted average of the price indices for Sana'a and Aden. The numbers for 2000 and 2001 are based on price data from all governorates. The numbers are end-of-period averages. 
Table 17. Republic of Yemen: Domestic Retail Prices for Petroleum Products and Electricity, 1998-2003

\begin{tabular}{|c|c|c|c|c|c|c|c|c|c|c|c|c|}
\hline & Dec. & Dec. & Dec. & Mar. & Jun. & Sep. & Dec. & Mar. & Jun. & Sep. & Dec. & Dec. \\
\hline & 1998 & 1999 & 2000 & \multicolumn{4}{|c|}{2001} & \multicolumn{4}{|c|}{2002} & 2003 \\
\hline & \multicolumn{12}{|c|}{ (In Yemeni rials per liter; unless otherwise indicated) } \\
\hline Gasoline & 35.0 & 35.0 & 35.0 & 35.0 & 35.0 & 35.0 & 35.0 & 35 & 35 & 35 & 35 & 35 \\
\hline Diesel & 10.0 & 10.0 & 17.0 & 17.0 & 17.0 & 17.0 & 17.0 & 17 & 17 & 17 & 17 & 17 \\
\hline Fuel oil & 11.0 & 13.0 & 25.0 & & $\ldots$ & $\ldots$ & $\ldots$ & $\ldots$ & $\ldots$ & $\ldots$ & $\ldots$ & $\ldots$ \\
\hline Kerosene & 15.0 & 16.0 & 16.0 & 16.0 & 16.0 & 16.0 & 16.0 & 16 & 16 & 16 & 16 & 16 \\
\hline Aviation fuel 1/ & 13.5 & 25.0 & $\ldots$ & $\ldots$ & $\ldots$ & $\ldots$ & $\ldots$ & $\ldots$ & $\ldots$ & $\ldots$ & $\ldots$ & $\ldots$ \\
\hline LPG (12.5 kg cylinder) & 200.0 & 200.0 & 200.0 & 220.0 & 220.0 & 220.0 & 220.0 & 220 & 220 & 220 & 220 & 220 \\
\hline \multirow[t]{2}{*}{ Electricity (per kwh) } & 8.70 & 8.70 & $\ldots$ & $\ldots$ & $\ldots$ & $\ldots$ & $\ldots$ & $\ldots$ & $\ldots$ & $\ldots$ & $\ldots$ & ... \\
\hline & \multicolumn{12}{|c|}{ (In U.S. dollars per liter; unless otherwise indicated) } \\
\hline Gasoline & 0.26 & 0.22 & 0.21 & 0.21 & 0.21 & 0.21 & 0.20 & 0.19 & 0.19 & 0.19 & 0.19 & 0.19 \\
\hline Diesel & 0.07 & 0.06 & 0.10 & 0.10 & 0.10 & 0.10 & 0.10 & 0.09 & 0.09 & 0.09 & 0.09 & 0.09 \\
\hline Fuel oil & 0.08 & 0.08 & 0.15 & $\ldots$ & $\ldots$ & $\ldots$ & $\ldots$ & $\ldots$ & $\ldots$ & $\ldots$ & $\ldots$ & $\ldots$ \\
\hline Kerosene & 0.11 & 0.10 & 0.10 & 0.10 & 0.10 & 0.09 & 0.09 & 0.09 & 0.09 & 0.09 & 0.09 & 0.09 \\
\hline Aviation fuel & 0.10 & 0.16 & $\ldots$ & $\ldots$ & $\ldots$ & $\ldots$ & $\ldots$ & $\ldots$ & $\ldots$ & $\ldots$ & $\ldots$ & $\ldots$ \\
\hline LPG (12.5 kg cylinder) & 1.46 & 1.25 & 1.22 & 1.33 & 1.31 & 1.29 & 1.27 & 1.2 & 1.2 & 1.2 & 1.2 & 1.19 \\
\hline Electricity (per kwh) & 0.06 & 0.05 & $\ldots$ & $\ldots$ & $\ldots$ & $\ldots$ & $\ldots$ & $\ldots$ & $\ldots$ & $\ldots$ & $\ldots$ & $\cdots$ \\
\hline \multicolumn{13}{|l|}{ Memorandum item: } \\
\hline $\begin{array}{l}\text { Exchange rate } \\
\text { (YRls/\$U.S.) 2/ }\end{array}$ & 137.0 & 160.5 & 164.0 & 166.0 & 168.0 & 170.0 & 173.3 & 174.0 & 175.6 & 177.1 & 179.0 & 184.3 \\
\hline
\end{tabular}

Sources: Ministry of Oil and Mineral Resources; and Fund staff estimates.

1/ In May 1999, aviation fuel prices were increased above world market levels and were to be maintained at levels exceeding international prices, which explains the increase implemented in April 2000.

2/ End-period free market exchange rate for the relevant month. 
Table 18. Republic of Yemen: Summary of Central Government Finance 1998-2003

\begin{tabular}{|c|c|c|c|c|c|c|}
\hline & 1998 & 1999 & 2000 & 2001 & 2002 & 2003 \\
\hline & \multicolumn{5}{|c|}{ (In millions of rials) } & \\
\hline Total revenue and grants & 225,959 & 338,253 & 603,680 & 568,401 & 588,790 & 672,870 \\
\hline Total revenue & 222,620 & 328,394 & 583,715 & 563,401 & 560,817 & 664,535 \\
\hline Oil and gas revenue & 117,772 & 210,598 & 376,033 & 406,166 & 391,237 & 480,191 \\
\hline Crude oil exports & 73,002 & 134,854 & 241,211 & 276,494 & 266,161 & 316,444 \\
\hline Domestic oil and gas gross & 44,770 & 75,744 & 134,822 & 129,672 & 125,076 & 153,747 \\
\hline Non-oil revenue & 104,848 & 117,796 & 207,682 & 157,234 & 169,580 & 184,344 \\
\hline Tax & 78,852 & 85,419 & 110,990 & 113,700 & 131,095 & 145,336 \\
\hline Nontax & 25,996 & 32,377 & 96,692 & 43,534 & 38,485 & 39,008 \\
\hline Grants (cash) & 3,339 & 9,859 & 19,965 & 5,000 & 27,973 & 8,335 \\
\hline Total expenditure and net lending & 280,080 & 340,872 & 480,531 & 527,083 & 609,436 & 778,214 \\
\hline Current expenditure & 227,496 & 281,376 & 397,928 & 406,011 & 484,980 & 581,154 \\
\hline Civilian wages and salaries & 64,287 & 78,341 & 98,173 & 111,227 & 134,592 & 143,238 \\
\hline Materials and services & 25,632 & 32,527 & 34,005 & 40,345 & 45,245 & 51,721 \\
\hline Defense (wage and nonwage) & 52,247 & 61,548 & 76,597 & 91,087 & 129,469 & 148,139 \\
\hline Interest obligations & 31,562 & 43,570 & 35,620 & 34,396 & 34,673 & 37,959 \\
\hline Domestic (net) & 22,459 & 32,260 & 29,166 & 26,799 & 26,171 & 29,181 \\
\hline External & 9,103 & 11,310 & 6,454 & 7,597 & 8,502 & 8,778 \\
\hline Explicit subsidies & 25,702 & 26,116 & 88,311 & 60,708 & 56,969 & 106,862 \\
\hline Wheat and flour & 25,702 & 4,329 & 0 & 0 & 0 & 0 \\
\hline Petroleum products & 0 & 21,787 & 88,311 & 60,708 & 52,469 & 100,862 \\
\hline Electricity: transfer to PEC & 0 & 0 & 0 & 0 & 4,500 & 6,000 \\
\hline Current transfers & 18,680 & 28,278 & 46,099 & 55,323 & 69,434 & 70,268 \\
\hline \multicolumn{7}{|l|}{ Of which } \\
\hline Public enterprises & 4,970 & 6,455 & 7,807 & 9,657 & 11,306 & 11,418 \\
\hline Social welfare fund & 4,388 & 5,946 & 10,574 & 10,717 & 18,679 & 18,669 \\
\hline Development $\mathrm{O}$ and $\mathrm{M}$ fund & 2,000 & 2,300 & 7,294 & 3,270 & 3,384 & 9,266 \\
\hline Other $1 /$ & 7,386 & 8,696 & 7,918 & 15,263 & 11,263 & 13,701 \\
\hline Development expenditure & 52,584 & 59,496 & 82,603 & 121,072 & 124,456 & 197,060 \\
\hline Net lending & 0 & 0 & 0 & 0 & 0 & 0 \\
\hline Overall balance (commitment) & $-54,121$ & $-2,619$ & 123,149 & 41,317 & $-20,646$ & $-105,344$ \\
\hline Rescheduled and pending interest obligations & 4,004 & 1,317 & -39 & 0 & 0 & 10,313 \\
\hline Domestic arrears & $-17,705$ & $-3,000$ & 0 & 0 & 0 & 0 \\
\hline Overall balance (cash) & $-67,822$ & $-4,302$ & 131,965 & 32,462 & 9,759 & $-95,031$ \\
\hline Financing & 67,865 & 441 & $-131,717$ & $-22,294$ & $-16,861$ & 80,349 \\
\hline External (net) & 10,089 & 24,668 & 10,488 & 4,120 & 9,665 & 15,795 \\
\hline Bilateral and multilateral loans (net) & $-8,654$ & 4,211 & $-8,940$ & 4,120 & 8,921 & 15,512 \\
\hline Disbursements & 21,980 & 36,060 & 21,650 & 23,291 & 26,201 & 31,118 \\
\hline Amortization obligations & $-30,634$ & $-31,850$ & $-30,590$ & $-19,170$ & $-17,281$ & $-15,605$ \\
\hline Amortization arrears & -553 & 0 & & 0 & $-7,151$ & 0 \\
\hline Rescheduled amortization & 19,297 & 20,458 & 9,580 & 0 & 7,896 & 0 \\
\hline Payment of late interest and short-term & & & & & & \\
\hline arrears (Paris Club and other) & 0 & 0 & 0 & 0 & 0 & 0 \\
\hline Domestic (net) & 57,776 & $-24,228$ & $-142,205$ & $-26,415$ & $-26,526$ & 101,465 \\
\hline Bank & 28,280 & $-46,888$ & $-171,205$ & $-48,323$ & $-23,998$ & $-14,700$ \\
\hline Central bank & 26,464 & $-50,616$ & $-194,390$ & $\ldots$ & $-44,423$ & 46,729 \\
\hline Commercial banks & 1,816 & 3,727 & 23,185 & & 20,425 & 37,190 \\
\hline Nonbank & 29,496 & 22,660 & 29,000 & 21,908 & $-2,528$ & 17,546 \\
\hline Discrepancy 2/ & 43 & $-3,861$ & 248 & 10,168 & $-7,102$ & $-14,700$ \\
\hline
\end{tabular}


Table 18. Republic of Yemen: Summary of Central Government Finance 1998-2003 (concluded)

\begin{tabular}{|c|c|c|c|c|c|c|}
\hline & 1998 & 1999 & 2000 & 2001 & 2002 & 2003 \\
\hline & \multicolumn{5}{|c|}{ (In percent of GDP) } & \\
\hline Overall balance (commitment) & -6.3 & -0.2 & 8.0 & 2.6 & -1.2 & -5.2 \\
\hline Overall balance (cash) & -7.9 & -0.4 & 8.6 & 2.0 & 0.6 & -4.7 \\
\hline Primary cash balance 3 / & -4.7 & 3.3 & 12.5 & 4.3 & 2.7 & -2.7 \\
\hline Public sector savings & -0.2 & 5.2 & 13.4 & 10.1 & 5.9 & 4.5 \\
\hline Total revenue and grants & 26.4 & 30.7 & 39.2 & 35.3 & 33.6 & 33.1 \\
\hline Total revenue & 26.0 & 29.8 & 37.9 & 35.0 & 32.0 & 32.6 \\
\hline Oil and gas revenue & 13.8 & 19.1 & 24.4 & 25.3 & 22.3 & 23.6 \\
\hline Crude oil exports & 8.5 & 12.2 & 15.7 & 17.2 & 15.2 & 15.5 \\
\hline Domestic oil and gas & 5.2 & 6.9 & 8.8 & 8.1 & 7.1 & 7.6 \\
\hline Non-oil revenue & 12.2 & 10.7 & 13.5 & 9.8 & 9.7 & 9.1 \\
\hline Tax revenue & 9.2 & 7.7 & 7.2 & 7.1 & 7.5 & 7.1 \\
\hline Nontax revenue & 3.0 & 2.9 & 6.3 & 2.7 & 2.2 & 1.9 \\
\hline Grants & 0.4 & 0.9 & 1.3 & 0.3 & 1.6 & 0.4 \\
\hline Total expenditure and net lending & 32.7 & 30.9 & 31.2 & 32.8 & 34.8 & 38.2 \\
\hline Current expenditure & 26.6 & 25.5 & 25.8 & 25.2 & 27.7 & 28.6 \\
\hline Civilian wages and salaries & 7.5 & 7.1 & 6.4 & 6.9 & 7.7 & 7.0 \\
\hline Materials and services & 3.0 & 3.0 & 2.2 & 2.5 & 2.6 & 2.5 \\
\hline Defense & 6.1 & 5.6 & 5.0 & 5.7 & 7.4 & 7.3 \\
\hline \multicolumn{7}{|l|}{ Of which } \\
\hline Wages and salaries & 3.8 & 3.6 & 3.3 & 3.2 & 3.3 & 3.3 \\
\hline Interest obligations & 3.7 & 4.0 & 2.3 & 2.1 & 2.0 & 1.9 \\
\hline Domestic (net) & 2.6 & 2.9 & 1.9 & 1.7 & 1.5 & 1.4 \\
\hline External & 1.1 & 1.0 & 0.4 & 0.5 & 0.5 & 0.4 \\
\hline Explicit subsidies & 3.0 & 2.4 & 5.7 & 3.8 & 3.2 & 5.2 \\
\hline Wheat and flour & 3.0 & 0.4 & 0.0 & 0.0 & 0.0 & 0.0 \\
\hline Petroleum & 0.0 & 2.0 & 5.7 & 3.8 & 3.0 & 5.0 \\
\hline Electricity & 0.0 & 0.0 & 0.0 & 0.0 & 0.3 & 0.3 \\
\hline Current transfers & 2.2 & 2.6 & 3.0 & 3.4 & 4.0 & 3.5 \\
\hline \multicolumn{7}{|l|}{ Of which } \\
\hline Public enterprises & 0.6 & 0.6 & 0.5 & 0.6 & 0.6 & 0.6 \\
\hline Social welfare fund & 0.5 & 0.5 & 0.7 & 0.7 & 1.1 & 0.9 \\
\hline Development expenditure & 6.1 & 5.4 & 5.4 & 7.5 & 7.1 & 9.7 \\
\hline Net lending & 0.0 & 0.0 & 0.0 & 0.0 & 0.0 & 0.0 \\
\hline Financing & 7.9 & 0.0 & -8.6 & -1.4 & -1.0 & 3.9 \\
\hline External (net) & 1.2 & 2.2 & 0.7 & 0.3 & 0.6 & 0.8 \\
\hline Domestic (net) & 6.7 & -0.4 & 0.0 & 0.6 & -0.4 & -0.7 \\
\hline Bank & 3.3 & -4.3 & -11.1 & -3.0 & -1.4 & -0.7 \\
\hline Central bank & 3.1 & -4.6 & -12.6 & $\ldots$ & -2.5 & 2.3 \\
\hline Commercial banks & 0.2 & 0.3 & 1.5 & $\cdots$ & 1.2 & 1.8 \\
\hline Nonbank & 3.4 & 2.1 & 1.9 & 1.4 & -0.1 & 0.9 \\
\hline Discrepancy 2/ & 0.0 & -0.4 & 0.0 & 0.6 & -0.4 & -0.7 \\
\hline
\end{tabular}

Sources: Ministry of Finance; Central Bank of Yemen; and Fund staff estimates.

1/ Allocations to the Agriculture and Roads Maintenance Funds, Development O and M, and other items.

2/ The discrepancy arises because some of the expenditure items are reported on a commitment basis, whereas the financing is recorded on a cash basis. The above- and below-the-line data will be reconciled, once the detailed information on the spending items committed but not disbursed becomes available.

3/ Overall balance, excluding interest obligations. 
Table 19. Republic of Yemen: Composition of Central Government

Revenues, 1998-2003

\begin{tabular}{|c|c|c|c|c|c|c|}
\hline & 1998 & 1999 & 2000 & 2001 & 2002 & 2003 \\
\hline & \multicolumn{5}{|c|}{ (In millions of Yemeni rials) } & \\
\hline Total revenue & 222,620 & 328,394 & 583,715 & 563,401 & 560,872 & 664,535 \\
\hline Oil revenue & 117,772 & 210,598 & 376,033 & 406,167 & 391,237 & 480,191 \\
\hline Crude oil export receipts & 73,002 & 134,854 & 241,211 & 276,494 & 266,161 & 316,444 \\
\hline Domestic oil and gas revenues & 44,770 & 75,744 & 134,822 & 129,672 & 125,076 & 163,747 \\
\hline Non-oil revenue & 104,848 & 117,796 & 207,682 & 157,234 & 169,580 & 184,344 \\
\hline Tax revenue & 78,852 & 85,419 & 110,990 & 113,700 & 131,095 & 145,335 \\
\hline Customs duties & 24,121 & 25,703 & 29,843 & 33,789 & 37,260 & 42,374 \\
\hline Taxes on goods and services & 24,974 & 24,419 & 36,944 & 27,918 & 29,998 & 32,351 \\
\hline Taxes on income & 13,093 & 16,389 & 22,246 & 46,864 & 34,215 & 36,183 \\
\hline Corporate profits tax & 13,156 & 14,498 & 18,444 & $\ldots$ & 22,523 & 26,224 \\
\hline Stamp taxes & 797 & $\ldots$ & 9 & 7 & 1 & $\ldots$ \\
\hline Zakat & 1,906 & 2,255 & 2,443 & 2,797 & 3,331 & 3,958 \\
\hline Real estate transfer tax & 757 & 943 & 953 & 1,203 & 1,013 & 1,359 \\
\hline Nontax revenue & 25,996 & 32,377 & 96,692 & 43,534 & 38,485 & 39,008 \\
\hline Profit transfers & 16,677 & 19,376 & 22,509 & 25,435 & 11,479 & 8,691 \\
\hline CBY & 13,000 & 13,750 & 14,900 & 16,952 & 10,000 & 7,557 \\
\hline Commercial banks & 12 & 53 & 381 & 100 & 234 & 494 \\
\hline Aden refinery & 200 & 10 & 2,775 & 276 & 145 & 340 \\
\hline YPC & 753 & 1,499 & $\ldots$ & 6,353 & 1,100 & 300 \\
\hline Non-oil nonfinancial public enterprises & 2,712 & 4,064 & 1,683 & 1,755 & 5,309 & 1,412 \\
\hline Cement & 1,079 & 1,329 & 600 & 718 & 1,168 & 506 \\
\hline Post and telecommunications & 574 & 700 & $\ldots$ & 88 & 3,787 & 468 \\
\hline Ports & 635 & 946 & 492 & 46 & 273 & 438 \\
\hline Airlines & 3 & 0 & $\ldots$ & $\ldots$ & 81 & $\ldots$ \\
\hline Other & 422 & 1,089 & 4,453 & 904 & 0 & $\ldots$ \\
\hline Fees and charges & 6,695 & $\ldots$ & 3,641 & 2,012 & 5,002 & 5,824 \\
\hline Construction & 357 & $\ldots$ & 340 & $\ldots$ & 73 & $\ldots$ \\
\hline Security & 2,019 & $\ldots$ & 1,857 & $\ldots$ & 2,451 & $\ldots$ \\
\hline Educational & 228 & $\ldots$ & 437 & $\ldots$ & 443 & $\ldots$ \\
\hline Foreign oil company exploration fees & 169 & $\ldots$ & 367 & 558 & 758 & 839 \\
\hline Vehicle fees & 530 & 596 & 8 & 634 & 676 & 739 \\
\hline Consular fees & 187 & 210 & 508 & 500 & 599 & 481 \\
\hline Delayed military service fee & 453 & 510 & 0 & 319 & 2 & 0 \\
\hline Other & 2,752 & $\ldots$ & 124 & $\ldots$ & 0 & $\ldots$ \\
\hline Other nontax revenue & 2,624 & $\ldots$ & $\ldots$ & 16,087 & 16,695 & $\ldots$ \\
\hline Fines and forfeitures & $\ldots$ & $\ldots$ & $\ldots$ & 2 & 9 & 8 \\
\hline Asset sales & 814 & $\ldots$ & $\ldots$ & $\ldots$ & 46 & $\ldots$ \\
\hline Foreign oil company signature bonuses & 882 & $\ldots$ & $\ldots$ & $\ldots$ & $\ldots$ & $\ldots$ \\
\hline \multirow[t]{2}{*}{ Other } & 928 & $\ldots$ & $\ldots$ & 434 & $\ldots$ & $\ldots$ \\
\hline & \multicolumn{5}{|c|}{ (In percent of GDP) } & \\
\hline Total revenue & 26.0 & 30.9 & 37.9 & 38.2 & 32.0 & 32.6 \\
\hline Oil revenue & 13.8 & 19.8 & 24.4 & 27.5 & 22.3 & 23.6 \\
\hline Crude oil exports & 8.5 & 12.7 & 15.7 & 18.8 & 15.2 & 15.5 \\
\hline Domestic oil and gas revenues & 5.2 & 7.1 & 8.8 & 8.8 & 7.1 & 8.0 \\
\hline Non-oil revenue & 12.2 & 11.1 & 13.5 & 10.7 & 9.7 & 9.1 \\
\hline Tax revenue & 9.2 & 8.0 & 7.2 & 7.7 & 7.5 & 7.1 \\
\hline Customs duties & 2.8 & 2.4 & 1.9 & 2.3 & 2.1 & 2.1 \\
\hline Taxes on goods and services & 2.9 & 2.3 & 2.4 & 1.7 & 1.7 & 1.6 \\
\hline Taxes on income & 1.5 & 1.5 & 1.4 & 2.9 & 2.0 & 1.8 \\
\hline Corporate profits tax & 1.5 & 1.4 & $\ldots$ & $\ldots$ & $\ldots$ & $\ldots$ \\
\hline
\end{tabular}


Table 19. Republic of Yemen: Composition of Central Government Revenues, 1998-2003 (concluded)

\begin{tabular}{lrrrrrr}
\hline & 1998 & 1999 & 2000 & 2001 & 2002 & 2003 \\
\hline & & & & & & \\
& & & & & \\
Nontax revenue & 3.0 & 3.0 & 6.3 & 3.0 & 2.2 & 1.9 \\
$\quad$ Profit transfers & 1.9 & 1.8 & 1.5 & 1.6 & 0.7 & 0.4 \\
$\quad$ Non-oil public enterprises & 0.3 & 0.4 & 0.1 & 0.1 & 0.3 & 0.1 \\
$\quad$ Fees and charges & 0.8 & $\ldots$ & 0.2 & 0.1 & 0.3 & 0.3 \\
$\quad$ Other nontax revenue & 0.3 & $\ldots$ & $\ldots$ & 1.0 & 1.0 & $\ldots$ \\
Memorandum item: & & & & & & \\
GDP at market prices (In millions of YRls) & 793,586 & $1,102,426$ & $1,539,386$ & $1,608,065$ & $1,753,505$ & $2,035,491$ \\
\hline
\end{tabular}

Sources: Ministry of Finance; Central Bank of Yemen; and Fund staff estimates. 
Table 20. Republic of Yemen: Composition of Central Government Expenditure, 1998-2003

\begin{tabular}{|c|c|c|c|c|c|c|}
\hline & 1998 & 1999 & 2000 & 2001 & 2002 & 2003 \\
\hline \multicolumn{7}{|c|}{ (In millions of Yemeni rials) } \\
\hline Total expenditure and net lending & 280,080 & 340,872 & 480,531 & 527,083 & 609,436 & 778,214 \\
\hline Current expenditures & 227,496 & 281,376 & 397,928 & 406,011 & 484,980 & 581,154 \\
\hline Civilian wages and salaries & 64,287 & 78,341 & 98,173 & 111,227 & 134,543 & 143,238 \\
\hline Materials and services & 25,632 & 32,527 & 34,005 & 40,345 & 45,245 & 51,721 \\
\hline Agricultural and road maintenance funds & 2,732 & $1,856 \ldots$ & & . & $3,384 \ldots$ & \\
\hline Defense & 52,247 & 61,548 & 76,597 & 91,087 & 129,469 & 148,139 \\
\hline Salaries & 32,464 & 38,659 & 43,552 & 50,750 & 51,008 & 67,643 \\
\hline Nonsalary & 19,783 & 22,889 & 33,045 & 40,337 & 78,461 & 80,496 \\
\hline Interest obligations & 31,562 & 43,570 & 35,620 & 34,396 & 34,673 & 37,959 \\
\hline Domestic (net until 1998) & 22,459 & 32,260 & 29,166 & 26,799 & 26,171 & 29,181 \\
\hline External & 9,103 & 11,310 & 6,454 & 7,597 & 8,502 & 8,778 \\
\hline Independent units & 4,654 & 6,841 & 7,918 & 9,656 & 11,262 & 13,710 \\
\hline Parliamentary and judiciary expenses & 4,023 & 5,945 & 6,742 & 1,544 & 9,648 & 11,910 \\
\hline Accounting and audit authority & 538 & 746 & 1,015 & 7,983 & 1,381 & 1,519 \\
\hline Election committee & 93 & 150 & 161 & 129 & 233 & 281 \\
\hline Explicit subsidies & 25,702 & 26,116 & 88,311 & 60,708 & 56,969 & 106,862 \\
\hline Wheat and flour & 25,702 & 4,329 & 0 & 0 & 0 & 0 \\
\hline Petroleum products & 0 & 21,787 & 88,311 & 60,708 & 52,469 & 100,862 \\
\hline Electricity; transfer to PEC & 0 & 0 & 0 & 0 & 4,500 & 6,000 \\
\hline Current transfers & 18,680 & 28,278 & 46,099 & 55,323 & 69,434 & 70,268 \\
\hline Public enterprises & 4,970 & 6,455 & 7,608 & 9,657 & 11,306 & 11,418 \\
\hline Social welfare & 6,709 & $\ldots$ & $\ldots$ & $\ldots$ & 56,128 & 54,015 \\
\hline \multicolumn{7}{|l|}{ Of which: } \\
\hline Social welfare fund & 4,388 & 5,946 & 10,574 & 10,717 & 18,679 & 18,669 \\
\hline Regular pension contributions & 2,736 & $\ldots$ & 9,479 & $\ldots$ & 13,053 & 7,193 \\
\hline Pension supplements & 0 & $\ldots$ & 694 & 4,283 & 970 & 1,037 \\
\hline Medical grants & 604 & $\ldots$ & $\ldots$ & $\ldots$ & 1181 & 1,108 \\
\hline Social and sports activities & $\ldots$ & $\ldots$ & $\ldots$ & $\ldots$ & 2343 & 118 \\
\hline Development $\mathrm{O}$ and $\mathrm{M}$ fund & 2,000 & 2,300 & 7,294 & 3,270 & 3,384 & 9,266 \\
\hline Development expenditure & 52,584 & 59,496 & 82,603 & 121,072 & 124,456 & 197,060 \\
\hline Net lending & 0 & 0 & 0 & 0 & 0 & 0 \\
\hline \multicolumn{7}{|l|}{ Memorandum item } \\
\hline GDP & 793,586 & $, 102,4261$, & $, 539,386$ & $, 608,065$ & $1,753,505$ & $2,035,491$ \\
\hline
\end{tabular}


Table 20. Republic of Yemen: Composition of Central Government Expenditure, 1998-2003 (concluded)

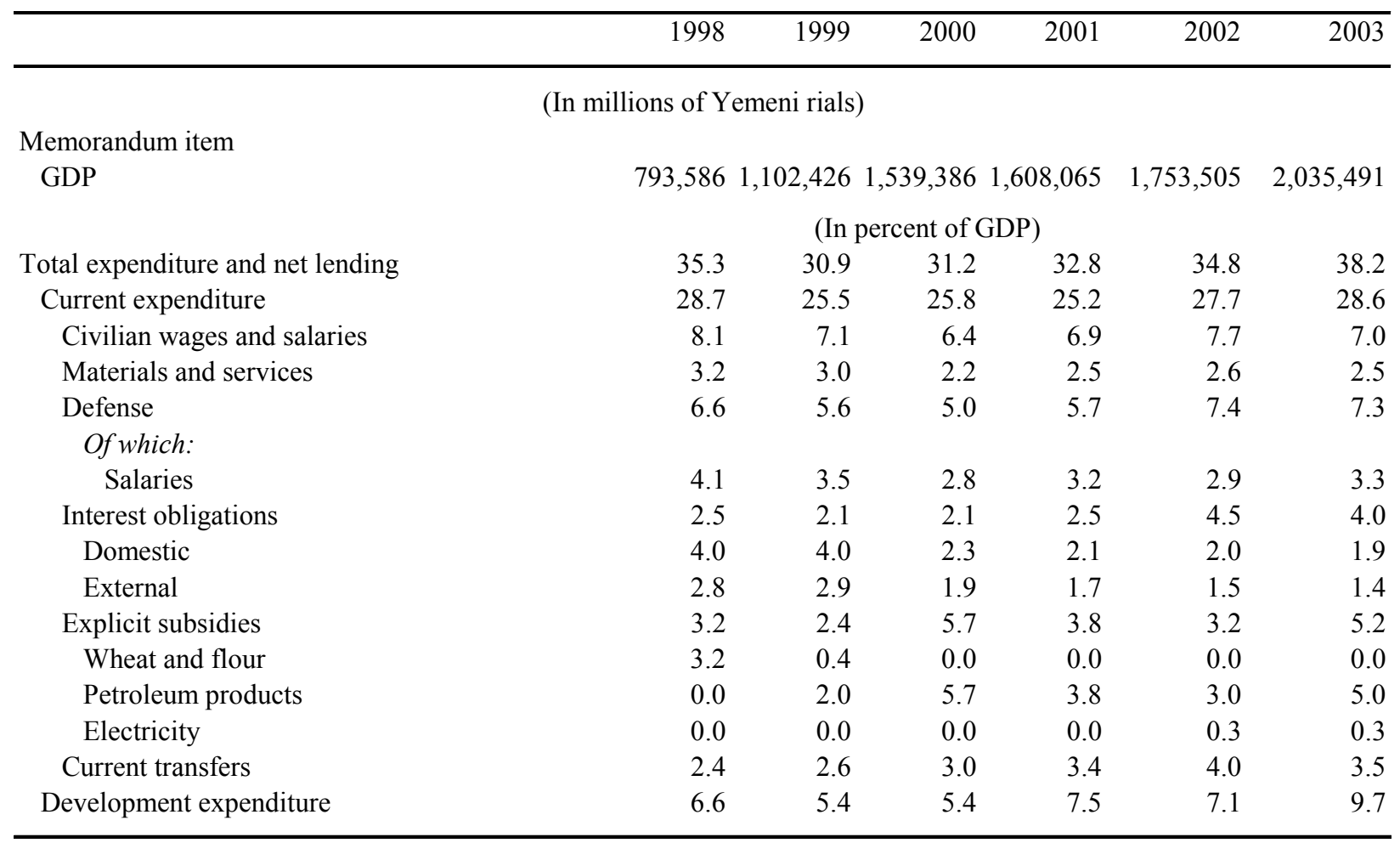

Sources: Ministry of Finance; Central Bank of Yemen; and Fund staff estimates. 
Table 21. Republic of Yemen: Current and Capital Transfers to Public Enterprises, 1998-2001

(In millions of Yemeni rials)

\begin{tabular}{lrrrr}
\hline & 1998 & 1999 & 2000 & 2001 \\
\hline Current transfers & 4,967 & 6,328 & 7,807 & 8,924 \\
Broadcasting and Television Corporation & 1,599 & 1,889 & 1,969 & 2,334 \\
Mineral Exploration Organization & 191 & 229 & 284 & $\ldots$ \\
Oil Exploration Corporation & 253 & 305 & 292 & 322 \\
Organization for Agricultural Research & 318 & 443 & 485 & 593 \\
Rural Development Organization & 140 & 169 & 176 & 313 \\
Al-Thawra Hospital & 781 & 1,214 & 1,258 & 1,589 \\
Touhama Development Organization & 198 & 234 & 267 & 302 \\
Yemen News Agency & 145 & 166 & 315 & 245 \\
Other agricultural and industrial enterprises & 1,030 & 1,324 & 2,181 & 3,122 \\
Financial support for troubled enterprises & 312 & 354 & 580 & 104 \\
Capital transfers & & & & \\
Broadcasting and Television Corporation & 15,135 & 14,661 & 21,092 & 29,770 \\
Civil Aviation and Meteorology Authority & 338 & 333 & 729 & 511 \\
National Water and Sewerage Corporation & 622 & 513 & 1,012 & 671 \\
Postal Service Organization & 3,009 & 3,012 & 4,085 & 5,286 \\
Public Authority for Rural Electricity and Water & 60 & 31 & 31 & 28 \\
Public Electricity Corporation & 2,587 & 3,012 & 4,920 & 7,581 \\
Rural Areas Development Organization & 7,201 & 6,536 & 8,085 & 12,408 \\
Al-Thawra Hospital & 115 & 85 & 393 & 547 \\
Yemen Company for Oil Investment in Jannah & 139 & 143 & 388 & 709 \\
Aden Free Zone Authority & 400 & 0 & 23 & 92 \\
Other & 107 & 79 & 107 & 354 \\
Total & 557 & 893 & 1,319 & 1,584 \\
\hline & & & & \\
& 20,102 & 20,989 & 28,899 & 38,694 \\
\hline
\end{tabular}

Source: Ministry of Finance. 
Table 22. Republic of Yemen: Monetary Survey, 1998-2004

\begin{tabular}{|c|c|c|c|c|c|c|c|}
\hline & $\frac{\text { Dec. }}{1998}$ & $\frac{\text { Dec. }}{1999}$ & $\frac{\text { Dec. }}{2000}$ & $\frac{\text { Dec. }}{2001}$ & $\frac{\text { Dec. }}{2002}$ & $\frac{\text { Dec. }}{2003}$ & $\frac{\text { Dec. }}{2004}$ \\
\hline Broad money & 333,350 & 379,294 & 474,525 & 563,050 & 664,664 & 797,372 & 917,275 \\
\hline Money & 179,927 & 207,197 & 247,248 & 282,683 & 306,450 & 347,465 & 390,541 \\
\hline Currency & 139,668 & 166,924 & 197,123 & 212,795 & 239,329 & 268,813 & 297,939 \\
\hline Demand deposits & 40,259 & 40,273 & 50,126 & 69,889 & 67,120 & 78,653 & 92,602 \\
\hline Quasi-money & 57,431 & 60,430 & 81,126 & 96,336 & 139,107 & 179,429 & 239,504 \\
\hline Foreign currency deposits & 95,992 & 111,668 & 146,151 & 184,030 & 219,108 & 270,478 & 287,230 \\
\hline Foreign assets (net) & 112,532 & 200,029 & 487,588 & 651,983 & 838,984 & 971,494 & $1,117,603$ \\
\hline Central bank (net) & 54,789 & 136,286 & 393,606 & 536,125 & 690,354 & 816,561 & 962,248 \\
\hline Assets & 138,927 & 237,687 & 482,478 & 636,989 & 792,163 & 923,527 & $1,057,492$ \\
\hline Liabilities & 84,138 & 101,401 & 88,872 & 100,864 & 101,809 & 106,966 & 95,244 \\
\hline Commercial banks (net) & 57,743 & 63,743 & 93,982 & 115,858 & 148,630 & 154,934 & 155,355 \\
\hline Assets & 64,857 & 70,991 & 103,340 & 120,511 & 151,981 & 158,925 & 165,128 \\
\hline Liabilities & 7,114 & 7,248 & 9,358 & 4,652 & 3,351 & 3,991 & 9,774 \\
\hline Domestic assets (net) & 220,818 & 179,266 & $-12,789$ & $-88,934$ & $-174,321$ & $-174,123$ & $-200,327$ \\
\hline Claims on government (net) & 219,768 & 173,468 & 1,331 & $-49,654$ & $-119,449$ & $-45,627$ & $-64,839$ \\
\hline Central bank (net) & 185,160 & 135,181 & $-59,209$ & $-100,771$ & $-147,023$ & $-100,294$ & $-166,409$ \\
\hline Claims & 201,724 & 159,621 & 17,913 & 2,082 & 686 & 206 & 2,606 \\
\hline Budget financing & 196,702 & 153,962 & 12,048 & 253 & 686 & 206 & 2,606 \\
\hline \multicolumn{8}{|l|}{ Counterpart to YBRD claims } \\
\hline Deposits & 16,564 & 24,440 & 77,122 & 102,853 & 147,709 & 100,500 & 169,015 \\
\hline Commercial banks (net) & 36,215 & 39,942 & 63,126 & 54,752 & 75,177 & 112,366 & 158,461 \\
\hline Claims & 36,267 & 39,972 & 63,173 & 54,814 & 75,215 & 112,467 & 158,680 \\
\hline Deposits & 52 & 30 & 46 & 62 & 38 & 101 & 219 \\
\hline $\begin{array}{l}\text { Total budget financing (net) } \\
\text { excluding YBRD }\end{array}$ & 216,352 & 169,464 & $-1,948$ & $-47,848$ & $-119,449$ & 12,072 & $-7,948$ \\
\hline \multicolumn{8}{|l|}{ Pension fund term deposits } \\
\hline at $\mathrm{CBY}$ & $-1,607$ & $-1,655$ & 2,586 & 3,635 & 47,602 & 57,699 & 56,892 \\
\hline Claims on nongovernment sector & 55,630 & 63,970 & 76,221 & 95,992 & 110,015 & 138,856 & 183,504 \\
\hline Private sector & 54,205 & 62,426 & 75,747 & 95,318 & 108,949 & 137,553 & 183,611 \\
\hline Rials & 40,212 & 42,948 & 43,995 & 54,246 & 64,275 & 73,534 & 101,471 \\
\hline Foreign currency & 17,242 & 23,999 & 31,751 & 41,072 & 44,674 & 64,019 & 82,140 \\
\hline Public enterprises & 1,120 & 895 & 0 & 0 & 270 & 289 & 0 \\
\hline Rials & 1,120 & 895 & 0 & 0 & 0 & 2 & 0 \\
\hline Foreign currency & 0 & 0 & 0 & 0 & 270 & 287 & 0 \\
\hline Mixed enterprises & 305 & 649 & 474 & 674 & 796 & 1,014 & 1,943 \\
\hline Valuation adjustment & $-13,231$ & $-9,194$ & $-17,665$ & $-37,888$ & $-74,714$ & $-124,378$ & $-151,781$ \\
\hline Other items (net) & $-41,349$ & $-48,978$ & $-72,950$ & $-97,383$ & $-90,173$ & $-142,974$ & $-167,210$ \\
\hline Capital and reserves & $-18,216$ & $-19,367$ & $-20,454$ & $-26,852$ & $-30,721$ & $-34,684$ & $-47,889$ \\
\hline Other (net) & $-23,133$ & $-29,611$ & $-52,496$ & $-70,531$ & $-59,452$ & $-108,291$ & $-119,321$ \\
\hline \multicolumn{8}{|l|}{ Memorandum item } \\
\hline Rial broad money & 237,358 & 267,627 & 328,374 & 379,019 & 445,556 & 526,894 & 630,046 \\
\hline
\end{tabular}


Table 22. Republic of Yemen: Monetary Survey, 1998-2004 (concluded)

\begin{tabular}{|c|c|c|c|c|c|c|c|}
\hline & $\frac{\text { Dec. }}{1998}$ & $\frac{\text { Dec. }}{1999}$ & $\frac{\text { Dec. }}{2000}$ & $\frac{\text { Dec. }}{2001}$ & $\frac{\text { Dec. }}{2002}$ & $\frac{\text { Dec. }}{2003}$ & $\frac{\text { Dec. }}{2004}$ \\
\hline Total net foreign currency position & 403.5 & 878.0 & $2,338.1$ & $3,039.4$ & $3,840.8$ & $4,388.8$ & $5,122.6$ \\
\hline Central Bank & 309.9 & 779.5 & $2,265.9$ & $2,966.5$ & $3,746.0$ & $4,257.5$ & $4,999.5$ \\
\hline \multicolumn{8}{|l|}{ Of which: } \\
\hline Net foreign assets & 386.7 & 853.3 & $2,289.3$ & $3,094.7$ & $3,859.3$ & $4,430.6$ & $5,177.8$ \\
\hline Foreign currency deposits & 76.7 & 73.8 & 112.0 & 127.6 & 113.3 & 173.1 & 178.3 \\
\hline Commercial banks & 93.6 & 98.5 & 72.2 & 72.9 & 94.8 & 131.2 & 123.0 \\
\hline \multicolumn{8}{|l|}{ Of which: } \\
\hline Net foreign assets & 407.5 & 399.1 & 547.0 & 668.7 & 830.9 & 840.7 & 838.2 \\
\hline Foreign currency deposits & 349.2 & 336.1 & 531.0 & 606.3 & 736.1 & 709.4 & 715.2 \\
\hline YBRD claims on Central Bank of Yemen & 35.4 & 35.4 & 34.1 & 10.6 & & 0.0 & 0.0 \\
\hline Official exchange rate: rials/US\$ (end-period) & 141.74 & 159.71 & 165.53 & 173.27 & 178.88 & 184.30 & 185.84 \\
\hline
\end{tabular}

Source: Central Bank of Yemen. 
Table 23. Republic of Yemen: Factors Affecting Domestic Liquidity, 1998-2004

(In millions of Yemeni rials)

\begin{tabular}{|c|c|c|c|c|c|c|c|}
\hline & 1998 & 1999 & 2000 & 2001 & 2002 & 2003 & 2004 \\
\hline & \multicolumn{7}{|c|}{ (Changes in end-year stocks) } \\
\hline Broad money 1/ & 34,962 & 45,944 & 95,231 & 88,525 & 101,615 & 132,707 & 119,904 \\
\hline Money & 13,543 & 27,269 & 40,052 & 35,435 & 23,766 & 41,016 & 43,076 \\
\hline Currency & 12,765 & 27,255 & 30,199 & 15,672 & 26,535 & 29,483 & 29,126 \\
\hline Demand deposits & 779 & 14 & 9,853 & 19,763 & $-2,768$ & 11,533 & 13,950 \\
\hline Quasi-money & 1,836 & 3,000 & 20,695 & 15,210 & 42,771 & 40,322 & 60,076 \\
\hline Foreign currency deposits $2 /$ & 19,582 & 15,676 & 34,484 & 37,879 & 35,078 & 51,369 & 16,752 \\
\hline Net foreign assets & $-32,645$ & 87,496 & 287,286 & 164,769 & 186,166 & 133,245 & 146,108 \\
\hline Central bank (net) & $-38,848$ & 81,497 & 257,047 & 142,893 & 153,394 & 126,942 & 145,687 \\
\hline Commercial banks (net) & 6,203 & 6,000 & 30,239 & 21,876 & 32,773 & 6,303 & 421 \\
\hline Net domestic assets 2/ & 67,606 & $-41,552$ & $-192,055$ & $-76,244$ & $-84,551$ & -538 & $-26,205$ \\
\hline Claims on government (net) & 55,611 & $-46,300$ & $-172,137$ & $-50,986$ & $-69,795$ & 73,822 & $-19,213$ \\
\hline Central bank (net) & 26,856 & $-49,979$ & $-194,390$ & $-41,562$ & $-46,252$ & 46,729 & $-66,115$ \\
\hline Budget financing (net) $3 /$ & 26,464 & $-50,616$ & $-194,596$ & $-37,526$ & $-44,423$ & 46,729 & $-66,115$ \\
\hline Commercial banks (net) & 1,816 & 3,727 & 23,184 & $-8,375$ & 20,425 & 37,190 & 46,095 \\
\hline \multicolumn{8}{|l|}{ Total budget financing (net) } \\
\hline excluding YBRD/pensions 4/ & 28,280 & $-46,888$ & $-171,412$ & $-45,900$ & $-23,998$ & 83,919 & $-20,020$ \\
\hline Claims on nongovernment sector & 19,559 & 8,340 & 12,251 & 9,627 & 23,567 & 28,791 & 45,298 \\
\hline Private sector & 19,825 & 8,221 & 13,321 & 19,571 & 13,631 & 28,604 & 46,058 \\
\hline Public enterprises & -383 & -225 & -895 & 0 & 270 & -270 & 0 \\
\hline Mixed enterprises & 117 & 344 & -174 & 200 & 2 & -2 & 0 \\
\hline Valuation adjustments 5/ & 501 & 3,969 & $-6,961$ & $-20,597$ & $-35,990$ & $-50,400$ & $-27,404$ \\
\hline Other items (net) & $-8,065$ & $-7,561$ & $-25,209$ & $-14,289$ & $-2,334$ & $-52,751$ & $-24,886$ \\
\hline Capital and reserves & $-12,367$ & $-5,033$ & 8,307 & $-6,397$ & $-3,869$ & $-3,963$ & $-13,205$ \\
\hline \multirow[t]{2}{*}{ Other } & 4,302 & $-2,528$ & $-33,516$ & $-7,891$ & 1,536 & $-48,788$ & $-11,681$ \\
\hline & \multicolumn{7}{|c|}{ (Changes in percent of broad money) } \\
\hline Broad money & 11.7 & 13.8 & 25.1 & 18.7 & 18.0 & 20.0 & 15.0 \\
\hline Money & 4.5 & 8.2 & 10.6 & 7.5 & 4.2 & 6.2 & 5.4 \\
\hline Currency & 4.3 & 8.2 & 8.0 & 3.3 & 4.7 & 4.4 & 3.7 \\
\hline Demand deposits & 0.3 & 0.0 & 2.6 & 4.2 & -0.5 & 1.7 & 1.7 \\
\hline Quasi-money & 0.6 & 0.9 & 5.5 & 3.2 & 7.6 & 6.1 & 7.5 \\
\hline Foreign currency deposits & 6.6 & 4.7 & 9.1 & 8.0 & 6.2 & 7.7 & 2.1 \\
\hline Net foreign assets & -10.9 & 26.2 & 75.7 & 34.7 & 33.1 & 20.0 & 18.3 \\
\hline Central bank (net) & -13.0 & 24.4 & 67.8 & 30.1 & 27.2 & 19.1 & 18.3 \\
\hline Commercial banks (net) & 2.1 & 1.8 & 8.0 & 4.6 & 5.8 & 0.9 & 0.1 \\
\hline Net domestic assets & 22.7 & -12.5 & -50.6 & -16.1 & -15.0 & -0.1 & -3.3 \\
\hline Claims on government (net) & 18.6 & -13.9 & -45.4 & -10.7 & -12.4 & 11.1 & -2.4 \\
\hline \multicolumn{8}{|l|}{ Of which } \\
\hline Central bank (net) 2/ & 9.0 & -15.0 & -51.3 & -8.8 & -8.2 & 7.0 & -8.3 \\
\hline Commercial banks (net) & 0.6 & 1.1 & 6.1 & -1.8 & 3.6 & 5.6 & 5.8 \\
\hline \multicolumn{8}{|l|}{ Total budget financing (net) } \\
\hline excluding YBRD/pensions 4/ & 9.5 & -14.1 & -45.2 & -9.7 & -4.3 & 12.6 & -2.5 \\
\hline
\end{tabular}


Table 23. Republic of Yemen: Factors Affecting Domestic Liquidity, 1997-2004 (concluded)

(In millions of Yemeni rials)

\begin{tabular}{|c|c|c|c|c|c|c|c|}
\hline & 1998 & 1999 & 2000 & 2001 & 2002 & 2003 & 2004 \\
\hline & \multicolumn{7}{|c|}{ (Changes in percent of broad money) } \\
\hline Claims on nongovernment sector & 6.6 & 2.5 & 3.2 & 2.0 & 4.2 & 4.3 & 5.7 \\
\hline Private sector & 6.6 & 2.5 & 3.5 & 4.1 & 2.4 & 4.3 & 5.8 \\
\hline Public enterprises & -0.1 & -0.1 & -0.2 & 0.0 & 0.0 & 0.0 & 0.0 \\
\hline Mixed enterprises & 0.0 & 0.1 & 0.0 & 0.0 & 0.0 & 0.0 & 0.0 \\
\hline Valuation adjustments & 0.2 & 1.2 & -1.8 & -4.3 & -6.4 & -7.6 & -3.4 \\
\hline Other items (net) & -2.7 & -2.3 & -6.6 & -3.0 & 0.0 & -7.9 & -3.1 \\
\hline Capital and reserves & -4.1 & -1.5 & 2.2 & -1.3 & -0.4 & -0.6 & -1.7 \\
\hline \multirow[t]{2}{*}{ Other } & 1.4 & -0.8 & -8.8 & -1.7 & 0.0 & -7.3 & -1.5 \\
\hline & \multicolumn{7}{|c|}{ (12-month changes in percent) } \\
\hline \multicolumn{8}{|l|}{ Memorandum items } \\
\hline Broad money 1/ & 11.7 & 13.8 & 25.1 & 18.7 & 18.0 & 20.0 & 15.0 \\
\hline Rial broad money & 6.9 & 12.8 & 22.7 & 15.4 & 17.6 & 18.3 & 19.6 \\
\hline Money & 8.1 & 15.2 & 19.3 & 14.3 & 8.4 & 13.4 & 12.4 \\
\hline Currency & 10.1 & 19.5 & 18.1 & 8.0 & 12.5 & 12.3 & 10.8 \\
\hline Rial demand deposits & 2.0 & 0.0 & 24.5 & 39.4 & -4.0 & 17.2 & 17.7 \\
\hline Rial quasi-money & 3.3 & 5.2 & 34.2 & 18.7 & 44.4 & 29.0 & 33.5 \\
\hline Foreign currency deposits & 25.6 & 16.3 & 30.9 & 25.9 & 19.1 & 23.4 & 6.2 \\
\hline Net foreign assets & -22.5 & 77.8 & 143.6 & 33.8 & 28.5 & 15.9 & 15.0 \\
\hline Central bank (net) & -41.5 & 148.7 & 188.6 & 36.3 & 28.6 & 18.4 & 17.8 \\
\hline Commercial banks (net) & 12.0 & 10.4 & 47.4 & 23.3 & 28.3 & 4.2 & 0.3 \\
\hline Net domestic assets 2/ & 44.1 & -18.8 & -107.1 & 596.2 & 95.0 & 0.3 & 15.0 \\
\hline Claims on government (net) & 33.9 & -21.1 & -99.2 & $-3,829.5$ & 140.6 & -61.8 & 42.1 \\
\hline Central bank (net) 2/ & 17.0 & -27.0 & -143.8 & 70.2 & 45.9 & -31.8 & 65.9 \\
\hline Commercial banks (net) & 5.3 & 10.3 & 58.0 & -13.3 & 37.3 & 49.5 & 41.0 \\
\hline Claims on nongovernment sector & 54.2 & 15.0 & 19.2 & 12.6 & 27.5 & 26.3 & 32.8 \\
\hline Private sector & 57.7 & 15.2 & 21.3 & 25.8 & 14.3 & 26.3 & 33.5 \\
\hline Rials & 63.3 & 6.8 & 2.4 & 23.3 & 18.5 & 14.4 & 38.0 \\
\hline Foreign currency & 43.5 & 39.2 & 63.0 & 29.4 & 8.8 & 43.3 & 28.3 \\
\hline \multirow[t]{2}{*}{ U.S. dollar equivalent } & 32.1 & 23.5 & 57.3 & 23.6 & 5.4 & 39.1 & 27.2 \\
\hline & \multicolumn{7}{|c|}{ (In percent of RYL broad money) } \\
\hline Currency & 0.59 & 0.62 & 0.60 & 0.59 & 0.54 & 0.51 & 0.47 \\
\hline Rial demand deposits & 0.17 & 0.15 & 0.15 & 0.15 & 0.15 & 0.15 & 0.15 \\
\hline Rial quasi-money & 0.24 & 0.23 & 0.25 & 0.26 & 0.31 & 0.34 & 0.38 \\
\hline
\end{tabular}

Source: Central Bank of Yemen (CBY).

1/ Includes deposits of public and private sector social security funds and deposits of public enterprises at central bank.

2/ Data incorporates valuation adjustments for foreign currency-denominated items.

3/ Total budget financing as used in the fiscal accounts includes budget financing (net) from the central bank and commercial bank net claims on government.

4/ YBRD: Yemen Bank of Reconstruction and Development.

5/ Applies to net foreign assets only. 
Table 24. Republic of Yemen: Balance Sheet of the Central Bank, 1998-2004

\begin{tabular}{|c|c|c|c|c|c|c|c|}
\hline End of period & $\frac{\text { Dec. }}{1998}$ & $\underline{\text { Dec. }}$ & $\frac{\text { Dec. }}{2000}$ & $\frac{\text { Dec. }}{2001}$ & $\frac{\text { Dec. }}{2002}$ & $\frac{\text { Dec. }}{2003}$ & $\frac{\text { Dec }}{2004}$ \\
\hline Foreign assets & 138,927 & 237,687 & 482,478 & 636,989 & 792,163 & 923,527 & $1,057,492$ \\
\hline Gold, silver, and foreign currency & 5,946 & 6,945 & 10,386 & 17,913 & 13,292 & 25,497 & 15,750 \\
\hline Foreign exchange held abroad & 107,635 & 192,319 & 454,391 & 611,442 & 770,327 & 859,272 & 948,676 \\
\hline Foreign securities & 3,436 & 10,180 & 3,672 & 3,802 & 564 & 37,451 & 83,127 \\
\hline SDR holdings & 21,910 & 28,243 & 14,029 & 3,832 & 7,981 & 1,308 & 9,940 \\
\hline $\begin{array}{l}\text { Claims on government } \\
\text { Of which: }\end{array}$ & 201,724 & 159,621 & 17,913 & 2,082 & 686 & 206 & 2,606 \\
\hline Budget financing & 196,702 & 153,962 & 12,048 & 253 & 686 & 206 & 2,606 \\
\hline Overdrafts & $\ldots$ & 141,108 & 0 & 0 & 0 & 0 & 0 \\
\hline Treasury bills & $\ldots$ & 12,854 & 12,048 & 253 & 686 & 206 & 2,606 \\
\hline Counterpart to YBRD claims $1 /$ & 5,022 & 5,659 & 5,865 & 1,829 & 0 & 0 & 0 \\
\hline (In millions of U.S. dollars) & 35 & 35 & 35 & 11 & 0 & 0 & 0 \\
\hline Claims on public enterprises & 1,108 & 895 & 0 & 0 & 0 & 0 & 0 \\
\hline Other assets & 17,340 & 24,652 & 19,983 & 23,429 & 16,890 & 17,047 & 19,892 \\
\hline Claims on commercial banks & 0 & 0 & 0 & 0 & 0 & 0 & 0 \\
\hline Fixed and other assets & 17,340 & 24,652 & 19,983 & 23,429 & 16,890 & 17,047 & 19,892 \\
\hline Assets $=$ Liabilities & 359,099 & 422,854 & 520,373 & 662,500 & 809,739 & 940,779 & $1,079,990$ \\
\hline $\begin{array}{l}\text { Foreign liabilities } \\
\text { Of which: }\end{array}$ & 84,138 & 101,401 & 88,872 & 100,864 & 10,809 & 106,966 & 95,244 \\
\hline AMF & 11,530 & 15,571 & 14,500 & 12,241 & 11,048 & 9,331 & 0 \\
\hline IMF & 47,464 & 65,233 & 52,320 & 64,791 & 67,678 & 72,852 & 69,377 \\
\hline Other & 25,144 & 20,597 & 22,052 & 23,832 & 23,084 & 24,784 & 25,867 \\
\hline Reserve money & 175,223 & 211,621 & 244,133 & 267,797 & 297,839 & 359,361 & 413,674 \\
\hline Currency outside banks & 139,668 & 166,924 & 197,123 & 212,795 & 239,329 & 268,813 & 297,939 \\
\hline Currency with banks & 2,663 & 2,160 & 4,318 & 4,398 & 4,446 & 5,460 & 4,859 \\
\hline Commercial banks deposits & 32,892 & 42,538 & 42,693 & 50,604 & 54,063 & 85,089 & 110,876 \\
\hline Rials & 14,899 & 20,564 & 27,392 & 31,963 & 29,321 & 34,145 & 56,430 \\
\hline Foreign currency & 17,993 & 21,974 & 15,301 & 18,642 & 24,743 & 50,944 & 54,446 \\
\hline Government deposits & 0 & 24,440 & 77,122 & 102,853 & 147,709 & 100,500 & 169,015 \\
\hline Rials (other government agencies) & 7,656 & 9,807 & 40,322 & 28,393 & 24,273 & 28,450 & 34,827 \\
\hline $\begin{array}{l}\text { Foreign currency (other government } \\
\text { agencies) }\end{array}$ & 8,908 & 14,633 & 28,493 & 27,910 & 71,992 & 57,526 & 76,498 \\
\hline $\begin{array}{l}\text { (In millions of U.S. dollars) } \\
\text { Rials (general government) }\end{array}$ & $\begin{array}{l}63 \\
\ldots\end{array}$ & $\begin{array}{l}92 \\
\ldots\end{array}$ & 172 & $\begin{array}{r}161 \\
46,551\end{array}$ & $\begin{array}{r}403 \\
42.032\end{array}$ & 312 & 412 \\
\hline Rials ( local Government) & $\cdots$ & $\cdots$ & $\cdots$ & $\ldots$ & 9,412 & $\cdots$ & $\ldots$ \\
\hline Public enterprise deposits & 27,754 & 22,556 & 27,653 & 43,008 & 37,569 & 54,208 & 46,015 \\
\hline Demand deposits & 8,769 & 10,624 & 9,122 & 20,897 & 17,302 & 22,306 & 22,975 \\
\hline Time deposits & 8,117 & 137 & 0 & 0 & 0 & 0 & 0 \\
\hline Foreign currency deposits & 10,868 & 11,795 & 18,531 & 22,112 & 20,267 & 31,902 & 23,040 \\
\hline (In millions of U.S. dollars) & 77 & 74 & 112 & 128 & 113 & 173 & 124 \\
\hline
\end{tabular}


Table 24. Republic of Yemen: Balance Sheet of the Central Bank, 1998-2004 (concluded)

\begin{tabular}{|c|c|c|c|c|c|c|c|}
\hline End of period & $\frac{\text { Dec. }}{1998}$ & $\frac{\text { Dec. }}{1999}$ & $\frac{\text { Dec. }}{2000}$ & $\frac{\text { Dec. }}{2001}$ & $\frac{\text { Dec. }}{2002}$ & $\frac{\text { Dec }}{200}$ & $\frac{\text { Dec }}{2004}$ \\
\hline Social security fund deposits & 1,607 & 1,655 & 2,586 & 3,635 & 47,602 & 57,699 & 56,892 \\
\hline In rials & 1,607 & 1,655 & 2,586 & 3,635 & 5,632 & 4,386 & 3,083 \\
\hline In U.S. dollars & 0 & 0 & 0 & 0 & 41,970 & 53,313 & 53,808 \\
\hline Certificates of deposit & 0 & 0 & 0 & 40,105 & 46,807 & 39,429 & 33,490 \\
\hline Banks & 0 & 0 & 0 & 29,961 & 46,807 & 38,779 & 31,440 \\
\hline Nonbanks & 0 & 0 & 0 & 10,144 & 0 & 650 & 2,050 \\
\hline Other liabilities & 53,813 & 61,181 & 80,007 & 104,238 & 130,405 & 222,616 & 265,660 \\
\hline SDR allocation & 5,723 & 6,300 & 6,208 & 6,253 & 6,948 & 7,841 & 8,284 \\
\hline Claims of YBRD 1/ & 5,022 & 5,659 & 5,865 & 1,829 & 0 & 0 & 0 \\
\hline Exchange valuation account & 13,231 & 9,194 & 17,665 & 37,888 & 74,714 & 124,378 & 151,781 \\
\hline Capital and reserves & 600 & 600 & 1,000 & 3,000 & 3,000 & 3,673 & 10,638 \\
\hline Other liabilities & 29,236 & 39,428 & 49,269 & 55,268 & 45,743 & 86,724 & 94,956 \\
\hline \multirow[t]{2}{*}{ Total liabilities } & 359,099 & 422,854 & 520,374 & 662,500 & 809,739 & 940,779 & $1,079,990$ \\
\hline & \multicolumn{7}{|c|}{ (In millions of U.S. dollars) } \\
\hline \multicolumn{8}{|l|}{ Memorandum items: } \\
\hline Net foreign assets & 387 & 853 & 2,376 & 3,095 & 3,855 & 4,431 & 5,178 \\
\hline Foreign currency deposits & 267 & 303 & 377 & 396 & 348 & 762 & 124 \\
\hline \multicolumn{8}{|l|}{ Of which: } \\
\hline Public enterprises & 77 & 74 & 112 & 128 & 113 & 173 & 124 \\
\hline Net foreign currency position & 270 & 740 & 2,227 & 2,931 & 2,966 & 4,215 & 5,009 \\
\hline Central bank gross foreign assets & 980 & 1,488 & 2,915 & 3,676 & 4,425 & 5,011 & 5,690 \\
\hline Central bank own gross foreign assets & 853 & 1,351 & 2,822 & 3,569 & 4,287 & 4,735 & 5,397 \\
\hline Multiplier (M2/reserve money) & 1.90 & 1.79 & 1.94 & 2.10 & 2.23 & 2 & 2 \\
\hline 12-month growth rate of reserve money & 13.2 & 20.8 & 15.4 & 9.7 & 11.2 & 21 & 15 \\
\hline
\end{tabular}

Source: Central Bank of Yemen.

1/ Foreign currency claims originally equivalent to US\$121 million valued at the free market exchange rate. Reduced by buybacks during 1997 and 2001. 
Table 25. Republic of Yemen: Consolidated Balance Sheet of Commercial Banks, 1998-2004 Commercial Banks, 1998-2004

\begin{tabular}{|c|c|c|c|c|c|c|c|c|}
\hline & $\frac{\text { Dec. }}{1997}$ & $\frac{\text { Dec. }}{1998}$ & $\underline{\text { Dec. }}$ & $\frac{\text { Dec. }}{2000}$ & $\frac{\text { Dec. }}{2001}$ & $\frac{\text { Dec. }}{2002}$ & $\frac{\text { Dec. }}{2003}-$ & $\frac{\text { Dec. }}{2004}$ \\
\hline ( In millions of Yemeni Rials) & \multicolumn{8}{|c|}{ ( In millions of Yemeni Rials) } \\
\hline Foreign assets & 59,472 & 64,857 & 70,991 & 103,340 & 120,511 & 151,981 & 158,925 & 165,128 \\
\hline Foreign currency & 3,914 & 3,877 & 4,206 & 4,953 & 6,057 & 8,880 & 10,626 & 8,748 \\
\hline Balances with banks abroad & 54,320 & 60,342 & 66,726 & 98,342 & 114,163 & 141,759 & 146,839 & 153,712 \\
\hline Foreign investments & & 638 & 59 & 45 & 291 & 1,339 & 1,459 & 2,668 \\
\hline Reserves & 28,052 & 35,714 & 43,631 & 45,545 & 50,647 & 55,710 & 87,593 & 112,679 \\
\hline Currency & 2,652 & 2,663 & 2,160 & 4,318 & 4,398 & 4,446 & 5,460 & 4,859 \\
\hline Deposits with central bank & 25,400 & 33,051 & 41,470 & 41,228 & 46,249 & 51,264 & 82,133 & 107,820 \\
\hline Rials & 15,592 & 15,283 & 19,912 & 26,422 & 27,859 & 27,661 & 32,830 & 53,610 \\
\hline Foreign currency & 9,808 & 17,768 & 21,558 & 14,805 & 18,389 & 23,603 & 49,303 & 54,210 \\
\hline (in millions of U.S. dollars) & 75 & 125 & 135 & 89 & 106 & 132 & 268 & 292 \\
\hline Certificates of deposit & 0 & 0 & 0 & 0 & 29,961 & 46,207 & 38,779 & 31,440 \\
\hline Claims on government & 34,873 & 36,267 & 39,972 & 63,173 & 54,814 & 75,215 & 112,467 & 158,680 \\
\hline Treasury bills & 34,549 & 36,267 & 39,972 & 63,173 & 54,814 & 75,215 & 112,467 & 158,680 \\
\hline Credit to government & 324 & 0 & 0 & 0 & 0 & 0 & 0 & 0 \\
\hline Claims on nongovernment sector & 39,544 & 54,521 & 63,075 & 76,221 & 95,992 & 110,015 & 138,856 & 185,554 \\
\hline Credit to public enterprises & 1,453 & 12 & 0 & 0 & 0 & 270 & 289 & 0 \\
\hline Rials & 1,257 & 12 & 0 & 0 & 0 & 0 & 2 & 0 \\
\hline Foreign currency & 196 & 0 & 0 & 0 & 0 & 270 & 287 & 0 \\
\hline Credit to private sector & 37,902 & 54,205 & 62,426 & 75,747 & 95,318 & 108,949 & 137,553 & 183,611 \\
\hline Rials & 24,631 & 40,212 & 42,948 & 43,995 & 54,246 & 64,275 & 73,534 & 101,471 \\
\hline Foreign currency & 9,858 & 17,242 & 23,999 & 31,751 & 41,072 & 44,674 & 64,019 & 82,140 \\
\hline Credit to mixed enterprises & 188 & 305 & 649 & 474 & 674 & 796 & 1,014 & 1,943 \\
\hline Other assets & 29,547 & 20,700 & 24,477 & 22,307 & 23,580 & 24,877 & 26,376 & 32,955 \\
\hline Interbranch accounts (net) & 151 & & 2 & & & & 502 & 852 \\
\hline YBRD claims on CBY 1/ & 4,630 & 5,022 & 5,659 & 5,865 & 1,829 & 0 & 0 & 0 \\
\hline Other assets & 24,767 & 15,677 & 18,816 & 16,441 & 21,751 & 24,877 & 25,873 & 32,103 \\
\hline Rials & 20,897 & 10,740 & 12,963 & 14,958 & 15,454 & 16,530 & 18,892 & 24,449 \\
\hline Foreign currency & 3,870 & 4,938 & 5,853 & 1,483 & 6,297 & 8,347 & 6,981 & 7,654 \\
\hline Total Assets & 191,487 & 212,059 & 242,146 & 310,585 & 375,503 & 464,004 & 562,996 & 686,436 \\
\hline \multicolumn{8}{|l|}{ Of which: } & 309,133 \\
\hline \multicolumn{9}{|l|}{ Liabilities } \\
\hline Foreign liabilities & 7,932 & 7,114 & 7,248 & 9,358 & 4,652 & 3,351 & 3,991 & 9,774 \\
\hline $\begin{array}{l}\text { Deposits of foreign banks } \\
\text { Of which: }\end{array}$ & 440 & 1,265 & 772 & 4,689 & 2,032 & 1,908 & 2,568 & 6,975 \\
\hline in local currency & 6 & 4 & 2 & 14 & 16 & 15 & 19 & 34 \\
\hline $\begin{array}{l}\text { Nonresidents deposits } \\
\text { Of which: }\end{array}$ & 2,041 & 950 & 947 & 852 & 791 & 1,442 & 1,423 & 2,799 \\
\hline in local currency & 1,131 & 318 & 250 & 429 & 146 & 156 & 199 & 232 \\
\hline Borrowing from foreign banks & 5,451 & 4,900 & 5,529 & 3,817 & 1,829 & 0 & 0 & 0 \\
\hline Demand deposits & 28,875 & 31,490 & 29,649 & 41,004 & 48,992 & 49,819 & 56,347 & 69,628 \\
\hline
\end{tabular}


Table 25. Republic of Yemen: Consolidated Balance Sheet of Commercial Banks, 1998-2004 Commercial Banks, 1998-2004 (concluded)

\begin{tabular}{|c|c|c|c|c|c|c|c|}
\hline & $\frac{\text { Dec. }}{1997}$ & $\frac{\text { Dec. }}{1998}$ & $\begin{array}{l}\text { Dec. } \\
999\end{array}$ & $\frac{\text { Dec. }}{2000}$ & $\frac{\text { Dec. }}{2001}$ & $\frac{\text { Dec. }}{002}$ & $\frac{\text { Dec }}{200}$ \\
\hline & \multicolumn{7}{|c|}{ ( In millions of Yemeni Rials) } \\
\hline Quasi-money deposits & 49,313 & 60,293 & 81,126 & 96,336 & 139,107 & 179,429 & 239,504 \\
\hline Time deposits & 24,924 & 30,427 & 41,782 & 50,320 & 78,492 & 106,947 & 148,234 \\
\hline Savings deposits & 20,705 & 25,681 & 34,652 & 40,952 & 51,582 & 59,573 & 72,775 \\
\hline Earmarked deposits & 3,684 & 4,185 & 4,692 & 5,064 & 9,034 & 12,909 & 18,495 \\
\hline Foreign currency deposits & 85,124 & 99,873 & 127,620 & 161,919 & 198,841 & 238,576 & 264,190 \\
\hline (In millions of U.S. dollars) & 601 & 625 & 771 & 934 & 1,112 & 1,295 & 1,422 \\
\hline Government deposits & 52 & 30 & 46 & 62 & 38 & 101 & 219 \\
\hline Rials & 47 & 26 & 44 & 61 & 37 & 98 & 80 \\
\hline \multicolumn{8}{|l|}{ Foreign currency } \\
\hline (In millions of U.S. dollars) & 5 & 4 & 2 & 1 & 1 & 3 & 139 \\
\hline Other liabilities & 38,966 & 45,052 & 51,432 & 63,542 & 72,849 & 84,553 & 103,122 \\
\hline Credit from central bank & 23 & 32 & 0 & 0 & 0 & 0 & 0 \\
\hline Rials & 23 & 0 & 0 & 0 & 0 & 0 & 0 \\
\hline Foreign currency & 0 & 32 & 0 & 0 & 0 & 0 & 0 \\
\hline Capital and reserves & 17,616 & 18,767 & 19,454 & 23,852 & 27,721 & 31,011 & 37,251 \\
\hline Interbranch accounts (net) & 82 & 0 & 136 & 2,072 & 424 & 0 & 0 \\
\hline Other liabilities & 21,246 & 26,255 & 31,841 & 37,618 & 44,705 & 53,542 & 65,872 \\
\hline Rials & 15,666 & 19,886 & 23,108 & 28,079 & 33,696 & 39,364 & 50,847 \\
\hline Foreign currency & 5,580 & 6,369 & 8,734 & 9,539 & 11,009 & 14,178 & 15,025 \\
\hline Total liabilities & 212,059 & 242,146 & 310,585 & 375,503 & 464,004 & 562,996 & 562,996 \\
\hline \multicolumn{8}{|l|}{ Of which: } \\
\hline In foreign currency & 97,501 & 113,273 & 145,270 & 175,949 & 213,030 & 256,530 & 256,530 \\
\hline \multicolumn{8}{|l|}{ Memorandum items: } \\
\hline Net foreign currency position & 87 & 93 & 72 & 70 & 89 & 125 & 124 \\
\hline Net foreign assets & 443 & 435 & 603 & 679 & 831 & 841 & 836 \\
\hline \multicolumn{8}{|l|}{ Foreign currency deposits (net) } \\
\hline in U.S. dollars & 356 & 342 & 531 & 609 & 742 & 716 & 716 \\
\hline \multicolumn{8}{|c|}{ (In percent of total assets) } \\
\hline Foreign assets & 30.6 & 29.3 & 33.3 & 32.1 & 32.8 & 28.2 & 24.1 \\
\hline Treasury bills and CDs & 17.1 & 16.5 & 20.3 & 22.6 & 26.2 & 26.9 & 27.7 \\
\hline Loans & 25.7 & 26.0 & 24.5 & 25.6 & 23.7 & 24.7 & 27.0 \\
\hline Reserves & 16.8 & 18.0 & 14.7 & 13.5 & 12.0 & 15.6 & 16.4 \\
\hline Other assets & 9.8 & 10.1 & 7.2 & 6.3 & 5.4 & 4.7 & 4.8 \\
\hline
\end{tabular}

Source: Central Bank of Yemen.

1/ YBRD: Yemen Bank of Reconstruction and Development 
Table 26. Republic of Yemen: Interest Rates, 1998-2004

\begin{tabular}{|c|c|c|c|c|c|c|c|}
\hline & 1998 & 1999 & 2000 & 2001 & 2002 & 2003 & 2004 \\
\hline \multicolumn{8}{|l|}{ Central bank lending rates } \\
\hline Government & 17.1 & 20.0 & 15.0 & 15.0 & 15.0 & $\ldots$ & $\ldots$ \\
\hline Public enterprises & 20.0 & 23.0 & 18.0 & 18.0 & 18.0 & $\ldots$ & $\ldots$ \\
\hline Commercial banks & 20.0 & 23.0 & 18.0 & 18.0 & 18.0 & $\ldots$ & $\ldots$ \\
\hline \multicolumn{8}{|l|}{ Commercial bank lending rates } \\
\hline Maximum rate & $\ldots$ & $\ldots$ & $\ldots$ & $\ldots$ & & & \\
\hline Rate range & $17-24$ & $23-28$ & $15-20$ & $15-20$ & $15-20$ & $15-21$ & $15-20$ \\
\hline \multicolumn{8}{|c|}{ Commercial bank (minimum/benchmark) } \\
\hline \multicolumn{8}{|l|}{ Deposit rates $2 /$} \\
\hline Savings deposits & 15.0 & 18.0 & 13.0 & 13.0 & 13.0 & 13.0 & 13.0 \\
\hline Three-month time deposits & $\ldots$ & $\ldots$ & $\ldots$ & $\ldots$ & $\ldots$ & $\ldots$ & $\ldots$ \\
\hline Six-month time deposits & $\ldots$ & $\ldots$ & $\ldots$ & $\ldots$ & $\ldots$ & $\ldots$ & $\ldots$ \\
\hline Nine-month time deposits & $\ldots$ & $\ldots$ & $\ldots$ & $\ldots$ & $\ldots$ & $\ldots$ & $\ldots$ \\
\hline One-year time deposits & $\ldots$ & $\ldots$ & $\ldots$ & $\ldots$ & $\ldots$ & $\ldots$ & $\ldots$ \\
\hline \multicolumn{8}{|c|}{ Specialized banks lending rates $3 /$} \\
\hline Short term & $\ldots$ & $\ldots$ & .. & $\cdots$ & $\ldots$ & $\ldots$ & $\ldots$ \\
\hline Medium and long term & $\ldots$ & $\ldots$ & $\ldots$ & $\ldots$ & $\ldots$ & $\ldots$ & $\ldots$ \\
\hline \multicolumn{8}{|c|}{$\begin{array}{l}\text { Treasury bill average interest rate on } \\
\text { successful bids }\end{array}$} \\
\hline Three-month & 17.5 & 18.1 & 13.3 & 12.9 & 10.9 & 14.0 & 14.6 \\
\hline Six-month & 17.7 & 17.4 & 13.6 & 12.5 & 11.2 & 13.9 & 14.7 \\
\hline Twelve-month & 17.8 & 16.2 & 12.9 & 12.1 & 11.3 & 13.9 & 14.6 \\
\hline
\end{tabular}

Source: Central Bank of Yemen.

1/ Prior to July 1995, a fixed interest rate structure applied to all loans and deposit rates. The mandated minimum deposit rates were not enforced and effective deposit rates were 3-5 percent. Interest rate reform in July 1995 freed interest rates, eliminated concessional loan rates, and enforced benchmark minimum commercial bank deposit rates.

2/ In May 1997, the benchmark system was narrowed to one minimum rate for savings accounts.

3/ As of June 1998, all specialized banks have adopted the interest rate structure applying to commercial banks. 


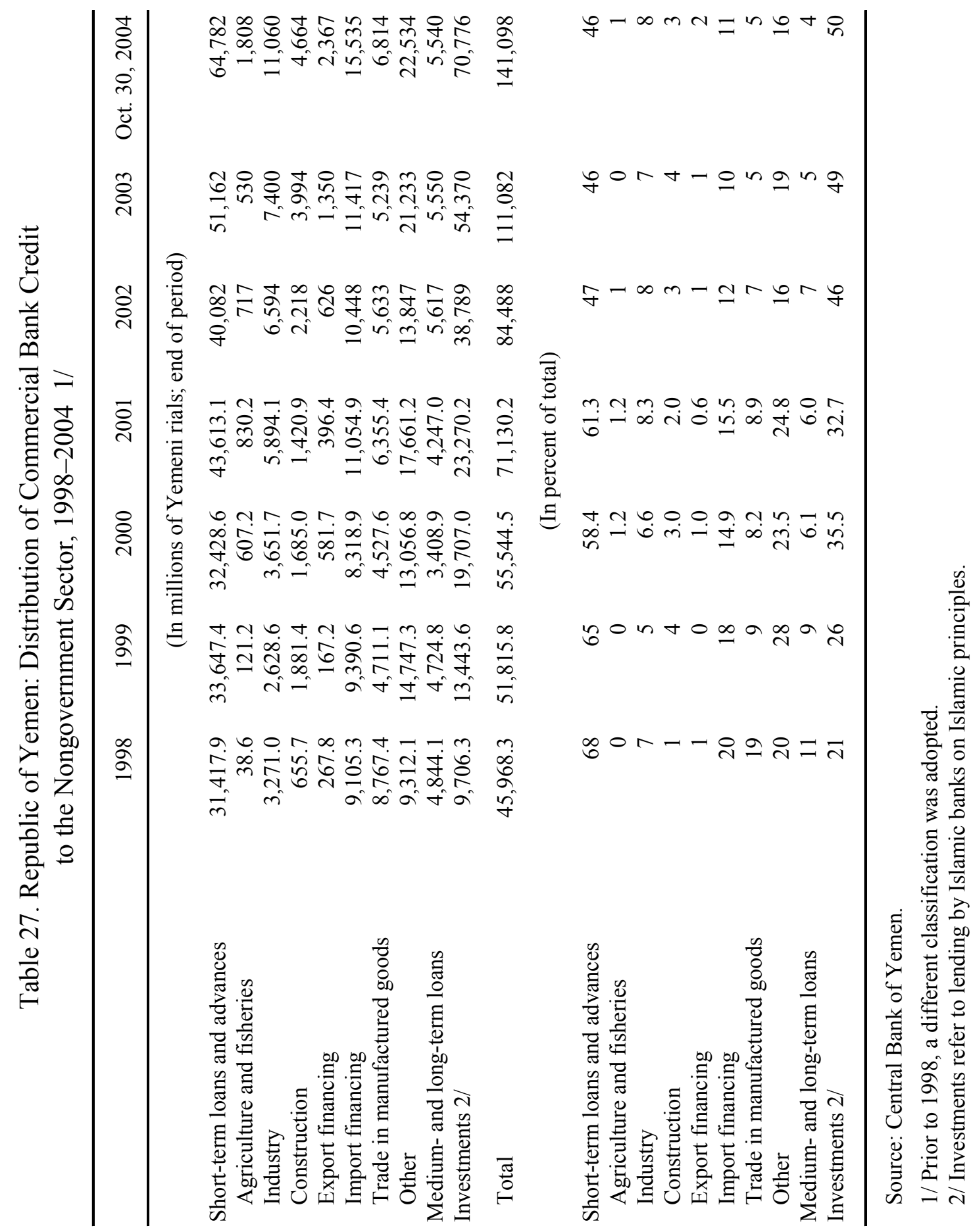


Table 28. Republic of Yemen: Commercial and Specialized Banks, at end-2004

\begin{tabular}{|c|c|c|c|c|c|c|}
\hline & $\begin{array}{c}\text { Date of } \\
\text { Establishment }\end{array}$ & $\begin{array}{c}\text { Head } \\
\text { Office } \\
\text { Location }\end{array}$ & $\begin{array}{c}\text { Capital } \\
\text { (Millions of } \\
\text { Yemeni Rials) }\end{array}$ & Shareholders & $\begin{array}{l}\text { Shareholding } \\
\text { (Percent) }\end{array}$ & $\begin{array}{c}\text { Total } \\
\text { Branches 1/ }\end{array}$ \\
\hline \multicolumn{7}{|l|}{ Commercial banks: } \\
\hline $\begin{array}{l}\text { Yemen Bank for Reconstruction } \\
\text { and Development (YBRD) }\end{array}$ & 1962 & Sana'a & $2,000.0$ & $\begin{array}{l}\text { Government } \\
\text { Yemeni private }\end{array}$ & $\begin{array}{l}51 \\
49\end{array}$ & 37 \\
\hline National Bank of Yemen & 1969 & Aden & $2,100.0$ & Government & 100 & 31 \\
\hline United Bank Ltd. & 1972 & Sana'a & $1,250.0$ & $\begin{array}{l}\text { Branch of United } \\
\text { Bank (Pakistan) }\end{array}$ & 100 & 2 \\
\hline Arab Bank PLC. & 1972 & Sana'a & $2,000.0$ & $\begin{array}{l}\text { Branch of Arab } \\
\text { Bank (Jordan) }\end{array}$ & 100 & 6 \\
\hline $\begin{array}{l}\text { Calyon Corporate and } \\
\text { Investment Bank }\end{array}$ & 1975 & Sana'a & $1,304.0$ & $\begin{array}{l}\text { Branch of Calyon } \\
\text { Corporate and } \\
\text { Investment Bank } \\
\text { (France) }\end{array}$ & 100 & 5 \\
\hline Yemen Commercial Bank & 1993 & Sana'a & $1,244.0$ & $\begin{array}{l}\text { Yemeni Private } \\
\text { Government }\end{array}$ & $\begin{array}{l}90 \\
10\end{array}$ & 8 \\
\hline $\begin{array}{l}\text { Yemen-Kuwait Bank for } \\
\text { Trade and Investment }\end{array}$ & 1979 & Sana'a & $1,250.0$ & Yemeni private & 100 & 5 \\
\hline International Bank of Yemen & 1979 & Sana'a & $1,250.0$ & $\begin{array}{c}\text { Yemeni private } \\
\text { Saudi Arabian Banks }\end{array}$ & $\begin{array}{l}80 \\
20\end{array}$ & 5 \\
\hline Rafidain Bank & 1982 & Sana'a & 60.0 & $\begin{array}{c}\text { Branch of Rafidain } \\
\text { Bank (Iraq) }\end{array}$ & 100 & 1 \\
\hline Yemen Bahrain Shamil Bank & 2002 & Sana'a & $2,000.0$ & $\begin{array}{l}\text { Yemeni private } \\
\text { Foreign private }\end{array}$ & $\begin{array}{l}75 \\
25\end{array}$ & 2 \\
\hline Yemen Gulf Bank & 2001 & Sana’a & $1,250.0$ & $\begin{array}{c}\text { Yemen Private } \\
\text { Foreign private } \\
\text { Private pension fund }\end{array}$ & $\begin{array}{c}77 \\
22 \\
1\end{array}$ & 2 \\
\hline \multicolumn{7}{|l|}{ Islamic banks: } \\
\hline $\begin{array}{l}\text { Islamic Bank for } \\
\text { Finance and Investment }\end{array}$ & 1995 & Sana'a & $1,250.0$ & $\begin{array}{c}\text { Yemeni private 2/ } \\
\text { Government } \\
\text { Foreign private }\end{array}$ & $\begin{array}{l}73.5 \\
4.5 \\
22.0\end{array}$ & 5 \\
\hline Altadamon Islamic Bank & 1996 & Sana'a & $3,000.0$ & $\begin{array}{l}\text { Yemeni private } 2 / \\
\text { Foreign private }\end{array}$ & $\begin{array}{c}96.7 \\
3.3\end{array}$ & 13 \\
\hline Saba Islamic Bank & 1997 & Sana'a & $1,975.0$ & $\begin{array}{l}\text { Yemeni private } 2 / \\
\text { Foreign private }\end{array}$ & $\begin{array}{l}85 \\
15\end{array}$ & 7 \\
\hline Al Watani & 1998 & Sana'a & $1,495.0$ & Yemeni private & 100 & 4 \\
\hline \multicolumn{7}{|l|}{ Specialized banks: } \\
\hline Housing Credit Bank & 1977 & Sana'a & 200.0 & $\begin{array}{l}\text { Government } \\
\text { Yemeni private }\end{array}$ & $\begin{array}{c}97 \\
3\end{array}$ & 2 \\
\hline $\begin{array}{l}\text { Cooperative and } \\
\text { Agricultural Credit }\end{array}$ & 1982 & Sana’a & 293.0 & $\begin{array}{c}\text { Government } \\
\text { Yemeni cooperatives }\end{array}$ & $\begin{array}{l}87 \\
13\end{array}$ & 34 \\
\hline
\end{tabular}

Source: Central Bank of Yemen.

$1 /$ Including head office.

2/ With some foreign minority shareholders. 
Table 29. Republic of Yemen: Indicators of Banking System Financial Soundness, 1999-2003 1/

(In percent)

\begin{tabular}{lrrrrr}
\hline & 1999 & 2000 & 2001 & 2002 & 2003 \\
\hline Portfolio quality: & & & & & \\
$\quad$ Problem loans/total loans & 44.0 & 34.0 & 31.0 & 33.0 & 29.0 \\
$\quad$ Problem loans/total assets & 11.7 & 9.0 & 8.0 & 8.0 & 7.3 \\
$\quad$ Provisions against problem loans/problem loans & 54.7 & 71.0 & 68.0 & 69.0 & 60.0 \\
$\quad$ Total capital and reserves/problem loans & 68.4 & 76.0 & 82.0 & 78.0 & 79.0 \\
Portfolio performance: & & & & & \\
$\quad$ Average return on assets & 1.0 & 1.0 & $\ldots$ & 1.0 & 1.0 \\
Average return on equity & 14.0 & 13.0 & $\ldots$ & 13.5 & 14.7 \\
Capital adequacy & & & & & \\
$\quad$ Total capital and reserves/total assets & & & & & \\
Risk-weighted capital adequacy ratio & 8.1 & 7.0 & 7.0 & 6.0 & 6.0 \\
Exposure to exchange rate risk & 5.2 & $\ldots$ & $\ldots$ & 10.0 & 11.0 \\
$\quad$ Total foreign currency assets (in billions of rials) & & & & & \\
Total foreign currency liabilities (in billions of rials) & 127.4 & 140.4 & 182.9 & 222.3 & 274.3 \\
Net exposure/total capital and reserves & 112.8 & 151.5 & 171.5 & 207.2 & 250.6 \\
Forex credits/forex deposits & 77.7 & 57.1 & 48.0 & 55.0 & 76.5 \\
Estimated exposure to real estate market & 19.0 & 24.8 & 25.3 & 24.3 & 26.8 \\
$\quad$ Total real estate loans/total loans & & & & & \\
Total construction loans/total loans & 5.2 & 4.0 & 3.8 & $\ldots$ & $\ldots$ \\
&
\end{tabular}

Source: Central Bank of Yemen.

1/ Data refer to the commercial banking system in aggregates and exclude specialized banks. 
Table 30. Republic of Yemen: Balance of Payments, 1998-2003

\begin{tabular}{|c|c|c|c|c|c|c|}
\hline & 1998 & 1999 & 2000 & 2001 & 2002 & 2003 \\
\hline & \multicolumn{6}{|c|}{ (In millions of U.S. dollars) } \\
\hline Trade balance & -725 & 24 & 1,169 & 531 & 502 & 502 \\
\hline Exports, f.o.b. & 1,501 & 2,464 & 3,805 & 3,302 & 3,584 & 3,923 \\
\hline Crude oil & 1,229 & 2,131 & 3,423 & 2,895 & 3,134 & 3,417 \\
\hline Government share & 471 & 993 & 1,944 & 1,531 & 1,562 & 1,741 \\
\hline Oil companies' share & 758 & 1,138 & 1,479 & 1,364 & 1,572 & 1,677 \\
\hline Oil products & 141 & 194 & 236 & 229 & 235 & 272 \\
\hline Non-oil exports & 132 & 140 & 146 & 178 & 215 & 234 \\
\hline Imports $1 /$ & $-2,226$ & $-2,440$ & $-2,635$ & $-2,771$ & $-3,083$ & $-3,422$ \\
\hline Oil products, f.o.b. & -117 & -174 & -232 & -206 & -212 & -245 \\
\hline Oil sector capital goods, c.i.f. & -66 & -71 & -215 & -242 & -295 & -296 \\
\hline Other imports, f.o.b. & $-2,043$ & $-2,195$ & $-2,188$ & $-2,323$ & $-2,576$ & $-2,881$ \\
\hline Food & -787 & -807 & -896 & -917 & -965 & -997 \\
\hline Nonfood & $-1,256$ & $-1,388$ & $-1,292$ & $-1,406$ & $-1,611$ & $-1,884$ \\
\hline Services, net & -388 & -503 & -630 & -718 & -633 & -748 \\
\hline Income & -312 & -630 & -674 & -579 & -718 & -827 \\
\hline Compensation of employees & -31 & -27 & 0 & 0 & 0 & 0 \\
\hline Oil company profits & -254 & -575 & -774 & -719 & -779 & -872 \\
\hline Current transfers & 1,254 & 1,314 & 1,400 & 1,273 & 1,384 & 1,201 \\
\hline General government transfers, net & 59 & 91 & 72 & 42 & 148 & 64 \\
\hline Workers' remittances, net & 1,191 & 1,223 & 1,228 & 1,231 & 1,230 & 1,137 \\
\hline Other transfers (oil signature bonus) & 4 & 0 & 100 & 0 & 6 & 0 \\
\hline Current account balance & -172 & 205 & 1,265 & 507 & 535 & 128 \\
\hline Capital account & 0 & 0 & 14 & 0 & 0 & 0 \\
\hline Financial account (excl. oil company transactions) & 79 & 414 & -43 & 246 & 81 & 430 \\
\hline Medium- and long-term loans, net & 20 & 68 & -46 & 94 & 53 & 126 \\
\hline Disbursements & $\ldots$ & $\ldots$ & 127 & 138 & 149 & 171 \\
\hline Amortization obligations & $\ldots$ & $\ldots$ & -173 & -45 & -96 & -46 \\
\hline Oil sector direct investment, net & -261 & -289 & -11 & 92 & 39 & -131 \\
\hline Private capital, net & 259 & 337 & 157 & 164 & 261 & 309 \\
\hline Errors and omissions & -95 & 159 & 174 & -84 & 97 & 156 \\
\hline Overall balance & -378 & 369 & 1,368 & 761 & 752 & 583 \\
\hline Financing & 378 & -369 & $-1,368$ & -761 & -752 & -583 \\
\hline Net reserves changes (increase -) & 246 & -508 & $-1,427$ & -762 & -752 & -583 \\
\hline Debt relief & 114 & 83 & 421 & 55 & 0 & 0 \\
\hline \multirow[t]{2}{*}{ Change in overdue obligations (increase + ) 2/ } & 32 & 56 & -363 & -54 & 0 & 0 \\
\hline & \multicolumn{6}{|c|}{ (In percent of GDP) } \\
\hline Current account & -2.8 & 2.7 & 13.2 & 5.3 & 5.4 & 1.1 \\
\hline Financial account & -3.0 & -0.6 & 2.0 & 4.1 & 3.0 & 2.2 \\
\hline \multirow[t]{2}{*}{ Overall balance } & -7.4 & 4.2 & 17.1 & 8.5 & 9.3 & 4.7 \\
\hline & \multicolumn{6}{|c|}{ (In millions of U.S. dollars; unless otherwise indicated) } \\
\hline Current account incl. grants (In percent of GDP) & -2.8 & 2.7 & 13.2 & 5.3 & 5.4 & 1.1 \\
\hline Of which: official grants (In percent of GDP) & 1.0 & 1.2 & 0.8 & 0.4 & 1.5 & 0.6 \\
\hline Central bank own gross foreign reserves 3 / & 853 & 1,351 & 2,822 & 3,569 & 4,056 & 4,445 \\
\hline (In months of imports) $3 /$ & 4.2 & 6.0 & 12.3 & 14.9 & 17.0 & 16.0 \\
\hline Official external debt 4/ & 5,281 & 5,490 & 4,832 & 4,870 & 4852 & 4978 \\
\hline (In percent of GDP) & 87.5 & 75.3 & 50.5 & 51.1 & 48.6 & 44.4 \\
\hline \multicolumn{7}{|l|}{ Debt service } \\
\hline (In percent of exports of goods and services) 4/ & 7.3 & 6.9 & 6.0 & 6.3 & 4.6 & 3.9 \\
\hline \multicolumn{7}{|l|}{ Change in outstanding Fund credit } \\
\hline (In millions of U.S. dollars; increase + ) & 84.5 & 73.5 & -73.8 & 44.4 & 31.0 & 29.1 \\
\hline
\end{tabular}

Sources: Central Bank of Yemen; and staff estimates.

1/ Imports are c.i.f. for current year and exclude imports of oil sector capital goods but include an adjustment for smuggling.

2/ Includes debt to non-Paris Club creditors under discussion to ensure comparable treatment.

3/ Includes central bank SDR holdings, foreign exchange held abroad, foreign securities, gold, silver, and foreign currencies; excludes commercial bank required foreign exchange reserves with the central bank against their foreign currency deposits.

4/ Public and publicly-guaranteed debt including central bank foreign liabilities. Debt and debt service reflect the 1996 and 1997 Paris Club reschedulings, including the 80 percent upfront discount provided by the Russian Federation and 2001 Paris Club stock of debt operation. 
Table 31. Republic of Yemen: Composition of Exports and Re-Exports, 1998-2003 1/

\begin{tabular}{|c|c|c|c|c|c|c|}
\hline & 1998 & 1999 & 2000 & 2001 & 2002 & 2003 \\
\hline & \multicolumn{6}{|c|}{ (In millions of Yemeni rials) } \\
\hline Food and live animals & 6,742 & 8,980 & 12,461 & 19,853 & 27,934 & 29,700 \\
\hline Beverages and tobacco & 530 & 783 & 1,005 & 846 & 1,238 & 2,712 \\
\hline Raw materials, inedible & 3,358 & 2,222 & 3,029 & 4,024 & 4,566 & 4,983 \\
\hline Mineral fuels and lubricants & 186,152 & 359,105 & 636,503 & 536,894 & 530,293 & 618,594 \\
\hline Of which: crude oil 2/ & 166,956 & 331,934 & 594,628 & 494,084 & 495,267 & 563,082 \\
\hline Of which: petroleum products $2 /$ & 19,196 & 27,171 & 41,875 & 42,810 & 35,026 & 55,512 \\
\hline Animals and vegetables oils and fat & 943 & 612 & 668 & 210 & 308 & 863 \\
\hline Chemicals & 857 & 1,015 & 2,136 & 1,863 & 1,882 & 2,132 \\
\hline Manufactures & 737 & 1,021 & 1,067 & 1,748 & 1,728 & 1,742 \\
\hline Machinery and transport equipment & 2,480 & 5,350 & 1,822 & 2,570 & 13,092 & 15,369 \\
\hline Miscellaneous manufactures & 1,489 & 901 & 1,138 & 969 & 4,889 & 8,803 \\
\hline Commodities not classified & 192 & 22 & 2 & 31 & 14 & 11 \\
\hline \multirow[t]{2}{*}{ Total, f.o.b. } & 203,480 & 380,011 & 659,831 & 569,008 & 585,946 & 684,908 \\
\hline & \multicolumn{6}{|c|}{ (In millions of U.S. dollars) 3/ } \\
\hline Food and live animals & 50 & 58 & 77 & 118 & 159 & 162 \\
\hline Beverages and tobacco & 4 & 5 & 6 & 5 & 7 & 15 \\
\hline Raw materials, inedible & 25 & 14 & 19 & 24 & 26 & 27 \\
\hline Mineral fuels and lubricants & 1,370 & 2,306 & 3,953 & 3,183 & 3,020 & 3,372 \\
\hline Of which: crude oil 2/ & 1,229 & 2,131 & 3,464 & 2,929 & 2,820 & 3,069 \\
\hline Of which: petroleum products $2 /$ & 141 & 174 & 490 & 254 & 199 & 303 \\
\hline Animals and vegetable oils and fat & 7 & 4 & 4 & 1 & 2 & 5 \\
\hline Chemicals & 6 & 7 & 13 & 11 & 11 & 12 \\
\hline Manufactures & 5 & 7 & 7 & 10 & 10 & 9 \\
\hline Machinery and transport equipment & 18 & 34 & 11 & 15 & 75 & 84 \\
\hline Miscellaneous manufactures & 11 & 6 & 7 & 6 & 28 & 48 \\
\hline Commodities not classified & 1 & 0.1 & 0.01 & 0.2 & 0.1 & 0.1 \\
\hline Total, f.o.b. & 1,497 & 2,440 & 4,098 & 3,373 & 3,336 & 3,733 \\
\hline
\end{tabular}

Sources: Central Statistics Organization; Central Bank of Yemen; and Ministry of Oil and Mineral Resources.

1/ Exports by SITC rev. 2 groups.

2/ Data provided by Ministry of Oil and Mineral Resources and Aden Refinery. Excludes reexports from Aden Refinery under processing arrangements and includes oil companies' share of crude oil exports.

3/ Converted from Yemeni rials at the average official exchange rate. 
Table 32. Republic of Yemen: Direction of Exports and Reexports, 1998-2003 1/ 2/

(In percent of total)

\begin{tabular}{|c|c|c|c|c|c|c|}
\hline & 1998 & 1999 & 2000 & 2001 & 2002 & 2003 \\
\hline Arab countries & 11.8 & 7.4 & 5.2 & 6.0 & 12.0 & 12.9 \\
\hline Djibouti & 0.7 & 0.2 & 0.2 & 0.2 & 0.3 & 0.2 \\
\hline Egypt & 2.0 & 0.3 & 0.1 & 0.3 & 0.4 & 0.3 \\
\hline Kuwait & 4.0 & 2.1 & 1.7 & 1.9 & 4.3 & 3.9 \\
\hline Saudi Arabia & 2.2 & 1.8 & 1.2 & 2.0 & 2.8 & 2.9 \\
\hline United Arab Emirates & 0.8 & 0.7 & 0.9 & 0.6 & 2.7 & 4.5 \\
\hline Other & 2.0 & 2.4 & 1.1 & 1.0 & 1.5 & 1.1 \\
\hline Africa & 2.6 & 0.3 & 0.9 & 3.5 & 3.0 & 2.2 \\
\hline Ethiopia & 0.2 & 0.2 & 0.1 & 0.1 & 0.9 & 0.1 \\
\hline South Africa & 2.4 & 0.0 & 0.7 & 3.3 & 1.7 & 1.0 \\
\hline Other & 0.0 & 0.1 & 0.1 & 0.1 & 0.4 & 1.1 \\
\hline Asia and Pacific & 74.2 & 88.3 & 82.5 & 76.0 & 77.4 & 80.3 \\
\hline China, People's Republic of & 24.9 & 27.6 & 19.0 & 10.8 & 15.1 & 31.2 \\
\hline India & 0.2 & 7.0 & 15.0 & 15.3 & 16.4 & 10.0 \\
\hline Japan & 3.7 & 1.0 & 2.1 & 0.4 & 1.4 & 1.1 \\
\hline Singapore & 5.9 & 8.0 & 5.5 & 4.6 & 3.9 & 4.3 \\
\hline South Korea & 11.3 & 13.5 & 17.3 & 21.1 & 12.2 & 5.3 \\
\hline Thailand & 21.7 & 23.1 & 17.2 & 18.0 & 18.6 & 21.5 \\
\hline Other & 6.5 & 8.1 & 6.5 & 5.8 & 9.8 & 6.9 \\
\hline European countries & 8.0 & 2.0 & 3.5 & 5.5 & 2.1 & 2.7 \\
\hline France & 1.5 & 0.1 & 0.3 & 1.9 & 0.1 & 0.3 \\
\hline Germany & 2.1 & 0.2 & 0.0 & 0.1 & 0.1 & 0.0 \\
\hline Italy & 0.8 & 0.2 & 0.8 & 2.0 & 0.3 & 0.3 \\
\hline Netherlands & 0.8 & 0.0 & 0.0 & 0.0 & 0.0 & 0.0 \\
\hline Spain & 0.1 & 0.1 & 0.0 & 0.1 & 0.1 & 0.3 \\
\hline United Kingdom & 0.4 & 0.2 & 0.1 & 0.0 & 0.2 & 0.2 \\
\hline Other & 2.2 & 1.3 & 2.3 & 1.3 & 1.3 & 1.6 \\
\hline American countries & 2.8 & 0.7 & 7.7 & 8.2 & 5.3 & 1.7 \\
\hline Brazil & 0.0 & 0.0 & 1.6 & 0.0 & 0.0 & 0.0 \\
\hline Canada & 0.1 & 0.1 & 0.0 & 0.1 & 0.0 & 0.0 \\
\hline United States & 2.7 & 0.6 & 6.1 & 8.1 & 5.3 & 1.7 \\
\hline Other & 0.0 & 0.0 & 0.0 & 0.0 & 0.0 & 0.0 \\
\hline Bunkering (ships) & 0.0 & 0.0 & 0.0 & 0.0 & 0.0 & 0.0 \\
\hline Bunkering (aircraft) & 0.0 & 0.0 & 0.0 & 0.0 & 0.0 & 0.0 \\
\hline Not classified & 0.5 & 1.3 & 0.3 & 0.8 & 0.2 & 0.2 \\
\hline Total, f.o.b. & 100.0 & 100.0 & 100.0 & 100.0 & 100.0 & 100.0 \\
\hline
\end{tabular}

Sources: Central Statistics Organization; Ministry of Oil and Mineral Resources; and staff estimates.

1/ Includes oil companies' share of crude oil exports.

2/ Excludes reexports from Aden Refinery under processing arrangements. 
Table 33. Republic of Yemen: Composition of Imports, 1998-2003 1/

\begin{tabular}{|c|c|c|c|c|c|c|}
\hline & 1998 & 1999 & 2000 & 2001 & 2002 & 2003 \\
\hline & \multicolumn{6}{|c|}{ (In millions of Yemeni rials) } \\
\hline Food and live animals & 83,667 & 103,400 & 111,573 & 120,721 & 126,851 & 158,939 \\
\hline Beverages and tobacco & 5,921 & 6,305 & 6,291 & 5,852 & 7,393 & 10,976 \\
\hline Raw materials, inedible & 6,605 & 6,584 & 8,158 & 10,681 & 11,232 & 14,449 \\
\hline Mineral fuels and lubricants & 18,977 & 25,165 & 44,931 & 50,022 & 75,173 & 100,203 \\
\hline Of which: petroleum products & 18,944 & 25,147 & 44,912 & 50,008 & 55,538 & 100,178 \\
\hline \multicolumn{7}{|l|}{ Animals and vegetable oils } \\
\hline and fat & 11,274 & 10,435 & 14,652 & 8,062 & 9,201 & 12,775 \\
\hline Chemicals & 28,690 & 29,123 & 40,857 & 38,379 & 44,450 & 52,932 \\
\hline Manufactures & 50,937 & 47,848 & 55,074 & 66,828 & 77,637 & 108,386 \\
\hline \multicolumn{7}{|l|}{ Machinery and transport } \\
\hline equipment & 71,357 & 67,251 & 78,323 & 95,244 & 134,835 & 177,938 \\
\hline Miscellaneous manufactures & 15,542 & 16,524 & 16,860 & 19,517 & 25,999 & 37,152 \\
\hline Commodities not classified & 1,540 & 114 & 3,816 & 593 & 253 & 379 \\
\hline \multirow[t]{2}{*}{ Total, f.o.b. } & 294,510 & 312,749 & 380,534 & 415,899 & 513,026 & 674,128 \\
\hline & \multicolumn{6}{|c|}{ (In millions of U.S. dollars) 2/ } \\
\hline Food and live animals & 616 & 664 & 693 & 716 & 722 & 866 \\
\hline Beverages and tobacco & 44 & 40 & 39 & 35 & 42 & 60 \\
\hline Raw materials, inedible & 49 & 42 & 51 & 63 & 64 & 79 \\
\hline Mineral fuels and lubricants & 140 & 162 & 279 & 297 & 428 & 546 \\
\hline Of which: petroleum products & 139 & 161 & 279 & 296 & 316 & 546 \\
\hline \multicolumn{7}{|l|}{ Animals and vegetable oils } \\
\hline and fat & 83 & 67 & 91 & 48 & 52 & 70 \\
\hline Chemicals & 211 & 187 & 254 & 228 & 253 & 289 \\
\hline Manufactures & 375 & 307 & 342 & 396 & 442 & 591 \\
\hline \multicolumn{7}{|l|}{ Machinery and transport } \\
\hline equipment & 525 & 432 & 486 & 565 & 768 & 970 \\
\hline Miscellaneous manufactures & 114 & 106 & 105 & 116 & 148 & 203 \\
\hline Commodities not classified & 11 & 1 & 24 & 4 & 1 & 2 \\
\hline Total, f.o.b. & 2,167 & 2,008 & 2,364 & 2,465 & 2,921 & 3,675 \\
\hline
\end{tabular}

Sources: Central Statistics Organization; Central Bank of Yemen; and Ministry of Oil and Mineral Resources.

1/ Imports by SITC rev. 2 groups.

2/ Converted from Yemeni rials at the average official exchange rate. 
Table 34. Republic of Yemen: Sources of Imports, 1998-2003 1/

(In percent of total)

\begin{tabular}{|c|c|c|c|c|c|c|}
\hline & 1998 & 1999 & 2000 & 2001 & 2002 & 2003 \\
\hline Arab countries & 32.1 & 38.3 & 37.2 & 37.6 & 40.6 & 40.2 \\
\hline Djibouti & 1.4 & 1.6 & 1.6 & 2.1 & 0.9 & 0.7 \\
\hline Egypt & 1.2 & 1.3 & 1.7 & 1.1 & 2.0 & 1.9 \\
\hline Kuwait & 2.3 & 5.7 & 4.7 & 5.2 & 7.4 & 7.9 \\
\hline Saudi Arabia & 11.7 & 11.6 & 13.8 & 12.4 & 12.0 & 11.1 \\
\hline United Arab Emirates & 9.0 & 12.1 & 9.9 & 12.5 & 13.0 & 14.1 \\
\hline Other & 6.5 & 5.9 & 5.5 & 4.3 & 5.3 & 4.5 \\
\hline Africa & 0.5 & 0.5 & 0.7 & 0.7 & 0.8 & 4.4 \\
\hline Ethiopia & 0.1 & 0.1 & 0.1 & 0.2 & 0.2 & 0.2 \\
\hline South Africa & 0.1 & 0.0 & 0.2 & 0.1 & 0.1 & 0.1 \\
\hline Other & 0.3 & 0.4 & 0.4 & 0.4 & 0.5 & 4.1 \\
\hline Asia and Pacific & 21.8 & 24.2 & 27.0 & 26.1 & 24.3 & 26.8 \\
\hline Australia & 2.4 & 4.2 & 2.5 & 1.5 & 1.1 & 0.8 \\
\hline China, People's Republic of & 3.1 & 3.0 & 3.5 & 3.9 & 5.9 & 5.4 \\
\hline India & 2.2 & 2.6 & 2.5 & 5.1 & 3.7 & 5.5 \\
\hline Japan & 3.4 & 2.6 & 3.1 & 2.2 & 2.1 & 2.5 \\
\hline Singapore & 3.2 & 3.4 & 3.1 & 1.5 & 1.0 & 1.1 \\
\hline Thailand & 1.3 & 1.9 & 3.2 & 1.8 & 2.0 & 2.2 \\
\hline Other & 6.2 & 6.5 & 9.1 & 10.1 & 8.5 & 9.3 \\
\hline European countries & 29.3 & 22.7 & 22.4 & 20.2 & 19.8 & 18.4 \\
\hline France & 5.6 & 4.2 & 4.5 & 4.7 & 3.5 & 3.5 \\
\hline Germany & 3.6 & 3.0 & 3.1 & 2.1 & 2.7 & 2.4 \\
\hline Italy & 4.3 & 3.5 & 2.6 & 1.5 & 1.6 & 1.6 \\
\hline Netherlands & 2.2 & 1.5 & 1.6 & 2.0 & 2.0 & 1.9 \\
\hline Spain & 0.5 & 0.2 & 0.2 & 0.7 & 0.4 & 0.4 \\
\hline United Kingdom & 5.1 & 4.1 & 3.2 & 3.8 & 3.3 & 3.1 \\
\hline Other & 8.1 & 6.1 & 7.2 & 5.4 & 6.3 & 5.5 \\
\hline American countries & 9.9 & 10.3 & 8.4 & 11.0 & 11.1 & 11.3 \\
\hline Brazil & 2.9 & 2.8 & 2.5 & 3.8 & 3.6 & 3.2 \\
\hline Canada & 0.6 & 0.8 & 0.3 & 0.5 & 0.7 & 0.8 \\
\hline United States & 5.8 & 5.9 & 4.4 & 4.9 & 5.7 & 6.3 \\
\hline Other & 0.7 & 0.8 & 1.2 & 1.8 & 1.1 & 1.0 \\
\hline Not classified & 0.3 & 0.2 & 0.6 & 0.8 & 3.4 & 0.2 \\
\hline Total, f.o.b. & 100.0 & 100.0 & 100.0 & 100.0 & 100.0 & 100.0 \\
\hline
\end{tabular}

Sources: Central Statistics Organization; Ministry of Oil and Mineral Resources; and Fund staff estimates.

1/ Excludes oil company investment goods imports. 
Table 35. Republic of Yemen: Enterprises Privatized/Liquidated Since 1997

\begin{tabular}{lll}
\hline Name & Trivatization Method & Timing \\
\end{tabular}

Industrial Sector

$\begin{array}{ll}\text { 1. Al-Thawra metal products } & 30 \text {-year lease. } \\ & \\ \text { 2. Agriculture and metal products } & 30 \text {-year lease. } \\ \text { 3. Leather shoe factory } & \begin{array}{l}\text { Land and building } \\ \text { restituted to original } \\ \text { owners. Equipment sold } \\ \text { and new land rented to } \\ \text { purchaser for } 30 \text { years. }\end{array}\end{array}$

4. Leather products cooperative Same as (3).

5. Martyrs’ garment factory

6. Vegetable oil factory

7. Soap factory

8. The public bakery

9. Women's sewing cooperative

10. Dairy factory (Aden)

11. The National company for sponge and metal furniture manufacturing Ltd.

Same as (3). leased for 30 years.

Equipment sold but no deal was concluded on leasing the site.

Equipment was sold and the building and land were rented for other purposes.

Sold to private sector.
Implemented in 1997 Lessee hires 35 of original staff and $\mathrm{MOF}^{1 /}$ continues to pay salaries/benefits of remaining workers.

Implemented in 1997, Lessee hires all of the effective 1/1/1998. 96 original staff.

Implemented in 1997, Purchaser hires 15 of effective 1/1/1998. original staff and MOF continues to pay salaries/benefits of remaining 78 employees.

Implemented in 1997. Purchaser keeps 5 staff and MOF continues to pay salaries/benefits of remaining 73 employees.

Implemented in 1997, Purchaser keeps 10 staff effective 1/1/1998. and MOF continues to pay salaries/benefits of remaining 98 .

Equipment sold and land

Implemented in 1998.

The 3 employees were retained by purchaser of equipment.

Sale of equipment $\mathrm{TPO}^{2 /}$ will take over in done in 1997. handling the fate of the site.

Sale of equipment Owner keeps 12 staff and finished in 1998, and MOF continues to pay land leased in 1999. salaries/benefits of remaining 75 employees.

Completed in 1999.

As of February 1998, had been tendered three times, very low bids each time. 
Table 35. Republic of Yemen: Enterprises Privatized/Liquidated Since 1997 (continued)

\begin{tabular}{llll}
\hline Name & Privatization Method & Timing & Remarks \\
\hline
\end{tabular}

12. Tomato paste factory (Lahaj) Liquidation.

Agricultural Sector

13. Hammam Ali State farm (Dhamar government)

14. Dar Sa'ad poultry (Aden government)

15. Old Fowah poultry (Hadramout)

16. New Fowah poultry (Hadramout)

17. Agricultural machinery rental (Station-Saiyoun Hadramout government)

18. Al-Mashhad farm (Hadramout)

19. Public corporation for foreign trade and grain

20. Hodeidah grain silos

Transport Sector

21. National Shipping Agency (Natship)

22. Stevedoring operations

Not available.
Labor force estimate in 1997: 118.
Privatized by rent.

Privatized by rent.

Privatized by rent.

Privatized by rent.

Privatized by management buyout under new name,

Saiyoun Company for

Agricultural and

Industrial Machinery.

Not available.

Liquidation.

Sold to private sector.

Not available.

Implemented in 1999.
This is the only enterprise that has been privatized through TPO.
Implemented in 1999.

Directly privatized by ministry. It was one of the two major activities (shipping) undertaken by the National Shipping Company.

Implemented in 1999 . Directly privatized by ministry. It was the second major activity (loading/ unloading cargoes) of the National Shipping Agency.

Process might not have been completed by yearend.
Implementation was still ongoing in 1999.

Supply and Trade Sector

\section{Consumption society for state, Liquidation.} public, and mixed sectors employees 
Table 35. Republic of Yemen: Enterprises Privatized/Liquidated Since 1997 (concluded)

\begin{tabular}{llll}
\hline Name & Privatization Method & Timing & Remarks \\
\hline $\begin{array}{l}\text { 24. Public corporation for building } \\
\text { material trade in Lahaj, } \\
\text { Abyan, Shabwah, and }\end{array}$ & Liquidation in progress. & Evaluation completed by \\
Hadramout. & & March 1999. \\
\hline
\end{tabular}

Source: The Yemeni authorities.

1/Minister of Finance.

2/Technical Privatisation Office. 